\title{
Confinement: Understanding the relation between the Wilson loop and dual theories of long distance Yang-Mills theory
}

\author{
M. Baker \\ University of Washington, Seattle, Washington 98105 \\ J. S. Ball \\ University of Utah, Salt Lake City, Utah 84112 \\ N. Brambilla and G. M. Prosperi \\ Dipartimento di Fisica dell'Universita-Milano, Istituto Nazionale di Fisica Nucleare, Sezione di Milano-Via Celoria 16, \\ 20133 Milano, Italy \\ F. Zachariasen \\ California Institute of Technology, Pasadena, California 91125 \\ (Received 4 March 1996)
}

\begin{abstract}
In this paper we express the velocity-dependent, spin-dependent heavy quark potential $V_{q \bar{q}}$ in QCD in terms of a Wilson loop $W(\Gamma)$ determined by pure Yang-Mills theory. We use an effective dual theory of longdistance Yang-Mills theory to calculate $W(\Gamma)$ for large loops, i.e., for loops of size $R>R_{\mathrm{FT}}$. [ $R_{\mathrm{FT}}$ is the flux tube radius, fixed by the value of the Higgs (monopole) mass of the dual theory, which is a concrete realization of the Mandelstam-'t Hooft dual superconductor mechanism of confinement.] We replace $W(\Gamma)$ by $W_{\text {eff }}(\Gamma)$, given by a functional integral over the dual variables, which for $R>R_{\mathrm{FT}}$ can be evaluated by a semiclassical expansion, since the dual theory is weakly coupled at these distances. The classical approximation gives the leading contribution to $W_{\text {eff }}(\Gamma)$ and yields a velocity-dependent heavy quark potential that for large $R$ becomes linear in $R$, and that for small $R$ approaches lowest-order perturbative QCD. This latter fact means that these results should remain applicable down to distances where radiative corrections giving rise to a running coupling constant become important. The spin dependence of the potential at long range as well as at short range reflects the vector coupling of quarks in QCD combined with the dual treatment of long-distance Yang-Mills theory. The methods developed here should be applicable to any realization of the dual superconductor mechanism. They give an expression determining $W_{\text {eff }}(\Gamma)$ independent of the classical approximation, but semiclassical corrections due to fluctuations of the flux tube are not worked out in this paper. Taking these into account should lead to an effective string theory free from the conformal anomaly. [S0556-2821(96)03916-1]

PACS number(s): 12.38.Aw, 11.15.Kc, 12.38.Lg, 12.39.Pn
\end{abstract}

\section{INTRODUCTION}

In this paper we give expressions for the heavy quark potential in QCD using an effective dual theory of longdistance Yang-Mills theory. This work goes beyond a previous treatment [1] where the quark motion was treated semiclassically and where the dual theory was considered only at the classical level, and provides an independent approach to the problem of the heavy quark potential.

In Sec. II we give the formulas for the heavy quark spindependent, velocity-dependent potential $V_{q \bar{q}}$ obtained in Refs. [2-4] in terms of a Wilson loop $W(\Gamma)$. This extends previous work of Eichten and Feinberg [5], Peskin [5], and others [6] to include the velocity-dependent spinindependent part of the potential. All momenta, spins, masses, and quantum mechanical properties of the quarks appear explicitly in the formulas relating $V_{q \bar{q}}$ to $W(\Gamma)$. The problem of the heavy quark potential is then reduced to calculating $W(\Gamma)$, a problem in pure Yang-Mills theory.

To clarify the techniques used in the dual description we illustrate them in Sec. III with the example of electrodynamics.
In Sec. IV we describe the dual theory and show how to calculate $W(\Gamma)$ for large loops. The size of the loop $\Gamma$ fixed by the classical trajectories of the moving quark-antiquark pair provides a length scale $R$ (the quark-antiquark separation) and we use the dual theory to evaluate $W(\Gamma)$ for $R>R_{\mathrm{FT}}$, the radius of the flux tube that forms between the moving quark-antiquark pair. This is done by replacing $W(\Gamma)$ by $W_{\text {eff }}(\Gamma)$, a functional integral over dual potentials $C_{\mu}$ that are the fundamental variables of the dual theory. We then obtain the spin-independent part of the heavy quark potential directly in terms of $W_{\text {eff }}(\Gamma)$. Finally we discuss the relation of the dual theory to recent work in $[7,8]$ on the use of electric-magnetic duality to determine the long-distance behavior of certain supersymmetric non-Abelian gauge theories.

In Sec. $\mathrm{V}$ we give explicit expressions for the spindependent part of the heavy quark potential in terms of quantities determined by the dual theory. Since the theory is weakly coupled at large distances, $W_{\text {eff }}(\Gamma)$ and, hence, $V_{q \bar{q}}$ can be evaluated by a semiclassical expansion.

In Sec. VI we calculate $W_{\text {eff }}(\Gamma)$ in the classical approximation to the dual theory. We show how these results yield 
the dual superconductor picture of confinement and discuss their relation to the "modified area law" model [9] for $W(\Gamma)$. Finally we remark how recent progress on quantization around classical vortex solutions [10] may be useful for calculating corrections to $W_{\text {eff }}(\Gamma)$ accounting for fluctuations of the length of the flux tube.

In Sec. VII we use the results of Sec. V and the classical solution to the dual theory to obtain the spin-dependent part of the heavy quark potential. This calculation gives a contribution to $V_{q}$ not obtained previously [11] and yields a simplified expression for the spin orbit potential that reflects the vector nature of both the short-range force and the confinement force.

In the conclusion we emphasize that, in contrast to Ref. [1], the methods developed in this paper yield an expression for $W_{\text {eff }}(\Gamma)$ valid independent of the classical approximation. Furthermore, we point out that the results presented here should be regarded more as consequences of the dual superconductor picture in general rather than of our particular realization of it [12].

\section{HEAVY QUARK POTENTIAL IN QCD}

To obtain the heavy quark potential $V_{q \bar{q}}$ [2], we make a Foldy-Wouthuysen transformation on the quark-antiquark Green's function and show that the result can be written as a Feynman path integral over particle and antiparticle coordinates and momenta of a Lagrangian depending only upon the spin, coordinates, and momenta of the quark and antiquark. Separating off the kinetic terms from this Lagrangian one can identify what remains as the heavy quark potential $V_{q \bar{q}}$. (Closed loops of light quark pairs and annihilation contributions were not included.) The terms in $V_{q \bar{q}}$ of order (quark mass) $^{-2}$ are of two types: velocity dependent $V_{\mathrm{VD}}$ and spin dependent $V_{\mathrm{SD}}$. The full potential $V_{q \bar{q}}$ is then

$$
V_{q \bar{q}}=V_{0}(R)+V_{\mathrm{VD}}+V_{\mathrm{SD}},
$$

where $V_{0}(R)$ is the static potential. These potentials are all expressed in terms of a Wilson loop $W(\Gamma)$ determined by pure Yang-Mills theory, given by

$$
W(\Gamma)=\frac{\int \mathcal{D} A e^{i S_{\mathrm{YM}}(A)} \operatorname{tr} P \exp \left(-i e \oint_{\Gamma} d x^{\mu} A_{\mu}(x)\right)}{\int \mathcal{D} A e^{i S_{\mathrm{YM}^{(}(A)}}} .
$$

The closed loop $\Gamma$ is defined by quark [antiquark] trajectories $\vec{z}_{1}(t)\left[\vec{z}_{2}(t)\right]$ running from $\vec{y}_{1}$ to $\vec{x}_{1}\left(\vec{x}_{2}\right.$ to $\left.\vec{y}_{2}\right)$ as $t$ varies from the initial time $t_{i}$ to the final time $t_{f}$. The quark [anti-quark] trajectories $\vec{z}_{1}(t)\left[\vec{z}_{2}(t)\right]$ define world lines $\Gamma_{1}\left(\Gamma_{2}\right)$ running from $t_{i}$ to $t_{f}\left(t_{f}\right.$ to $\left.t_{i}\right)$. The world lines $\Gamma_{1}$ and $\Gamma_{2}$, along with two straight lines at fixed time connecting $\vec{y}_{1}$ to $\vec{y}_{2}$ and $\vec{x}_{1}$ to $\vec{x}_{2}$, then make up the contour $\Gamma$ (see Fig. 1). As usual $A_{\mu}(x)=\frac{1}{2} \lambda_{a} A_{\mu}^{a}(x)$, tr means the trace over color indices, $P$ prescribes the ordering of the color matrices according to the direction fixed on the loop and $S_{\mathrm{YM}}(A)$ is the Yang-Mills action including a gauge fixing term. We have denoted the Yang-Mills coupling constant by $e$, i.e.,

$$
\alpha_{s}=\frac{e^{2}}{4 \pi} \text {. }
$$

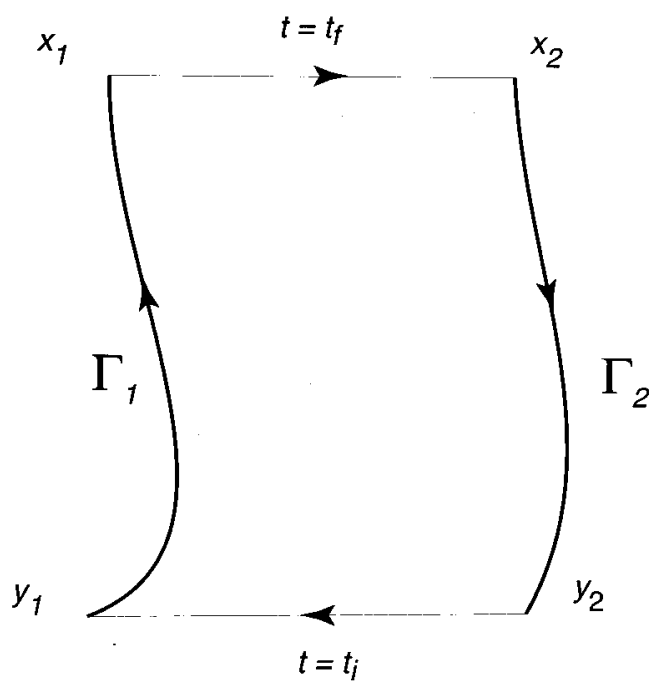

FIG. 1. Wilson loop for the quark-antiquark system.

The spin-independent part of the potential, $V_{0}+V_{\mathrm{VD}}$, is obtained from the zero order and the quadratic terms in the expansion of $i \ln W(\Gamma)$ for small velocities $\vec{z}_{1}(t)$ and $\vec{z}_{2}(t)$. This expansion has the form

$$
\begin{aligned}
i \ln W(\Gamma)= & \int_{t_{i}}^{t_{f}} d t\left(V_{0}(R(t))\right. \\
& \left.+\sum_{i, j=1}^{2} \sum_{k, \ell=1}^{3} \dot{z}_{i}^{k}(t) V_{i j}^{k \ell}(\vec{R}(t)) \dot{z}_{j}^{\ell}(t)\right),
\end{aligned}
$$

where $\vec{R}(t)=\vec{z}_{1}(t)-\vec{z}_{2}(t)$, and

$$
V_{\mathrm{VD}}=\sum_{i, j=1}^{2} \sum_{k, \ell=1}^{3} \dot{z}_{i}^{k}(t) V_{i j}^{k \ell}(\vec{R}(t)) \dot{z}_{j}^{\ell}(t) .
$$

$[i \ln W(\Gamma)$ has an expansion of the form (2.4) only to second order in the velocities.] The expression (2.5) for $V_{\mathrm{VD}}$ follows from the same argument used to identify $V_{0}(R)$ as the velocity-independent term in the expansion (2.4). We can write Eq. (2.4) in the form

$$
i \ln W(\Gamma)=-\int_{t_{i}}^{t_{f}} d t L_{I}\left(z_{1}, z_{2}, \dot{z}_{1}, \dot{z}_{2}\right),
$$

where

$$
-L_{I}=V_{0}(R)+V_{\mathrm{VD}}
$$

is an effective interaction Lagrangian for classical particles moving along trajectories $\vec{z}_{1}(t)$ and $\vec{z}_{2}(t)$ with gauge couplings $e(-e)$ and we can then interpret $i \ln W(\Gamma)$ as an effective action describing the motion of classical particles after elimination of the Yang-Mills field.

The spin-dependent potential $V_{\mathrm{SD}}$ contains structures for each quark analogous to those obtained by making a FoldyWouthuysen transformation on the Dirac equation in an external field $F_{\mu \nu}^{\mathrm{ext}}$ along with an additional term $V_{\mathrm{SS}}$ having the structure of a spin-spin interaction. We can then write 


$$
V_{\mathrm{SD}}=V_{\mathrm{LS}}^{\mathrm{mag}}+V_{\text {Thomas }}+V_{\text {Darwin }}+V_{\mathrm{SS}}
$$

using a notation that indicates the physical significance of the individual terms (mag denotes magnetic). The first two terms in Eq. (2.8) can be obtained by making the replacement

$$
F_{\mu \nu}^{\mathrm{ext}}(x) \rightarrow\left\langle\left\langle F_{\mu \nu}(x)\right\rangle\right\rangle,
$$

in the corresponding expression for the interaction of a Dirac particle in an external field, where

$$
\langle\langle f(A)\rangle\rangle \equiv \frac{\int \mathcal{D} A e^{i S_{Y M}(A)} \operatorname{tr} P\left\{\exp \left[-i e \oint_{\Gamma} d x^{\mu} A_{\mu}(x)\right] f(A)\right\}}{\int \mathcal{D} A e^{i S_{Y M}(A)} \operatorname{tr} P \exp \left[-i e \oint_{\Gamma} d x^{\mu} A_{\mu}(x)\right]},
$$

and

$$
F_{\mu \nu}=\partial_{\mu} A_{\nu}-\partial_{\nu} A_{\mu}-i e\left[A_{\mu}, A_{\nu}\right]
$$

i.e., $\left\langle\left\langle F_{\mu \nu}(x)\right\rangle\right\rangle$ is the expectation of the Yang-Mills field tensor in the presence of a quark and antiquark moving along classical trajectories $\vec{z}_{1}(t)$ and $\vec{z}_{2}(t)$, respectively.

The explicit expressions for $V_{\mathrm{LS}}^{\mathrm{mag}}$ and $V_{\text {Thomas }}$ obtained in Ref. [2] are $^{1}$

$$
\int d t V_{\mathrm{LS}}^{\mathrm{mag}}=\sum_{j=1}^{2} \frac{e}{m_{j}} \int_{\Gamma_{j}} d x^{\sigma} S_{j}^{\ell}\left\langle\left\langle\hat{F}_{\ell \sigma}(x)\right\rangle\right\rangle
$$

and

$$
\int d t V_{\text {Thomas }}=-\sum_{j=1}^{2} \frac{e}{2 m_{j}^{2}} \int_{\Gamma_{j}} d x^{\mu} S_{j}^{\ell} \epsilon^{\ell k r} p_{j}^{k}\left\langle\left\langle F_{\mu r}(x)\right\rangle\right\rangle,
$$

where

$$
\hat{F}_{\mu \nu}=-\frac{1}{2} \varepsilon_{\mu \nu \rho \sigma} F^{\rho \sigma}
$$

$\vec{S}_{j}$ is the spin matrix, and $m_{j}$ is the mass of the $j$ th quark. Because the expression for $V_{\text {Thomas }}$ contains an explicit factor of $1 / m_{j}^{2}$ the integral over the trajectory of the $j$ th quark $\int_{\Gamma_{j}} d x^{\mu}\left\langle\left\langle F_{\mu r}(x)\right\rangle\right\rangle$ can be replaced by $(-1)^{j+1} \int_{t_{i}}^{t_{f}} d t\left\langle\left\langle F_{0 r}\left(z_{j}\right)\right\rangle\right\rangle$ evaluated for static quarks. This gives the usual expression for $V_{\text {Thomas }}$ in terms of the derivative of the static potential (see Sec. V). The expression for $V_{\mathrm{LS}}^{\mathrm{mag}}$ on the other hand contains only a single power of $1 / m_{j}$ and $\int_{\Gamma_{j}} d x x^{\sigma}\left\langle\left\langle\hat{F}_{\ell \sigma}(x)\right\rangle\right\rangle$ must be evaluated to first order in the quark velocities. There results the usual magnetic interaction of the spin of the $j$ th quark with the expectation value $\left\langle\left\langle\hat{F}_{\ell \sigma}\left(z_{j}\right)\right\rangle\right\rangle$.

The expression for $V_{\text {Darwin }}$ is

\footnotetext{
${ }^{1}$ Here and in the following $\int_{\Gamma_{j}} d x^{\mu} f_{\mu}(x)$ $\equiv(-1)^{j+1} \int_{t_{i}}^{t_{f}} d t\left[f_{0}\left(z_{j}\right)-\vec{z}_{j} \cdot \vec{f}\left(z_{j}\right)\right]$, where $z_{j}=\left(t, \vec{z}_{j}(t)\right)$. The factor $(-1)^{j+1}$ accounts for the fact that world line $\Gamma_{2}$ runs from $t_{f}$ to $t_{i}$. We also use the notation $z_{j}^{\prime}=\left(t^{\prime}, \vec{z}_{j}\left(t^{\prime}\right)\right)$.
}

$$
\int d t V_{\text {Darwin }}=-\sum_{j=1}^{2} \frac{e}{8 m_{j}^{2}} \int_{\Gamma_{j}} d x^{\mu}\left\langle\left\langle D^{\nu} F_{\nu \mu}(x)\right\rangle\right\rangle,
$$

where

$$
D^{\nu} F_{\nu \mu}=\partial^{\nu} F_{\nu \mu}-i e\left[A^{\nu}, F_{\nu \mu}\right] \text {. }
$$

Again because of the explicit factor of $1 / \mathrm{m}_{j}^{2}$, the integral over the trajectory $\Gamma_{j}$ of the $j$ th quark is evaluated for static quarks.

The final term $V_{\mathrm{SS}}$ in Eq. (2.8) is given by

$$
\begin{aligned}
\int V_{\mathrm{SS}} d t= & -\frac{1}{2} \sum_{j, j^{\prime}=1}^{2} \frac{i e^{2}}{m_{j} m_{j^{\prime}}} T_{s} \int_{\Gamma_{j}} d x^{\mu} \\
& \times \int_{\Gamma_{j^{\prime}}} d x^{\prime}{ }^{\sigma} S_{j}^{\ell} S_{j^{\prime}}^{k}\left(\left\langle\left\langle\hat{F}_{\ell \mu}(x) \hat{F}_{k \sigma}\left(x^{\prime}\right)\right\rangle\right\rangle\right. \\
& \left.-\left\langle\left\langle\hat{F}_{\ell \mu}(x)\right\rangle\right\rangle\left\langle\left\langle\hat{F}_{k \sigma}\left(x^{\prime}\right)\right\rangle\right\rangle\right),
\end{aligned}
$$

where $T_{s}$ is the spin time ordering operator along the paths $\Gamma_{1}$ and $\Gamma_{2}$, and the averages are evaluated for static quarks. The terms $j \neq j^{\prime}$ in Eq. (2.17) give a spin-spin interaction proportional to $1 / m_{1} m_{2}$ while the terms $j=j^{\prime}$ in Eq. (2.17) give a spin-independent term proportional to $\left(1 / m_{1}^{2}+1 / m_{2}^{2}\right)$. The spin ordering is relevant only for these latter terms.

We have thus obtained the explicit expression (2.8) for the spin-dependent potential as a sum of terms depending upon the quark and antiquark spins, masses, and momenta with coefficients that are expectation values $\langle\langle\rangle\rangle$ of operators computed in Yang-Mills theory in the presence of classical sources generated by the moving quark-antiquark pair. We now show that these expectation values can be obtained as functional derivatives of $i \ln W(\Gamma)$ with respect to the path, i.e., with respect to the trajectories $\vec{z}_{1}(t)$ or $\vec{z}_{2}(t)$. For example, consider the change in $W(\Gamma)$ induced by letting

$$
\vec{z}_{1}(t) \rightarrow \vec{z}_{1}(t)+\delta \vec{z}_{1}(t), \quad \text { where } \delta \vec{z}_{1}\left(t_{i}\right)=\delta \vec{z}_{1}\left(t_{f}\right)=0
$$

Then from the definitions (2.2) and (2.10), it follows that

$$
\delta i \ln W(\Gamma)=-e \int_{t_{i}}^{t_{f}} \frac{\delta S^{\mu \nu}\left(z_{1}\right)}{2}\left\langle\left\langle F_{\mu \nu}\left(z_{1}\right)\right\rangle\right\rangle,
$$

where

$$
\delta S^{\mu \nu}\left(z_{1}\right)=\left(d z_{1}^{\mu} \delta z_{1}^{\nu}-d z_{1}^{\nu} \delta z_{1}^{\mu}\right) .
$$

Equation (2.18) then gives

$$
-e\left\langle\left\langle F_{\mu \nu}\left(z_{1}\right)\right\rangle\right\rangle=\frac{\delta i \ln W(\Gamma)}{\delta S^{\mu \nu}\left(z_{1}\right)}
$$

and similarly one can get

$$
e\left\langle\left\langle F_{\mu \nu}\left(z_{2}\right)\right\rangle\right\rangle=\frac{\delta i \ln W(\Gamma)}{\delta S^{\mu \nu}\left(z_{2}\right)} .
$$

Varying the path $\vec{z}_{2}(t)$ in Eq. (2.20) gives 


$$
\begin{aligned}
& e^{2}\left(\left\langle\left\langle F_{\mu \nu}\left(z_{1}\right) F_{\rho \sigma}\left(z_{2}\right)\right\rangle\right\rangle-\left\langle\left\langle F_{\mu \nu}\left(z_{1}\right)\right\rangle\right\rangle\left\langle\left\langle F_{\rho \sigma}\left(z_{2}\right)\right\rangle\right\rangle\right) \\
& \quad=i e \frac{\delta}{\delta S^{\rho \sigma}\left(z_{2}\right)}\left\langle\left\langle F_{\mu \nu}\left(z_{1}\right)\right\rangle\right\rangle .
\end{aligned}
$$

The first and second variational derivatives of $W(\Gamma)$ then determine the expectation values of $F_{\mu \nu}$ needed to evaluate $V_{\mathrm{SD}}$. Furthermore, we show in an appendix that $\left\langle\left\langle D^{\nu} F_{\nu \mu}(x)\right\rangle\right\rangle$ appearing in $V_{\text {Darwin }}$ can also be expressed in terms of variational derivatives of $W(\Gamma)$. The Wilson loop $W(\Gamma)$ that is determined by pure Yang-Mills theory then fixes the complete heavy quark potential $V_{q \bar{q}}$. Thus, up to order (1/quark mass) ${ }^{2}$ the dynamics of a quark anti-quark pair in QCD is completely fixed by the dynamics of YangMills theory. The properties of the quark spins, masses, etc., appear only as given kinematic factors in the terms defining the heavy quark potential.

The structure (2.8)-(2.21) of $V_{\mathrm{SD}}$ is a consequence of the vector nature of the QCD interaction just as the structure of the Fermi-Breit potential is a consequence of the vector nature of the QED interaction. The latter potential results from the $(1 / \mathrm{mass})^{2}$ expansion of the electron positron Green function with a corresponding omission of closed loops of electron positron pairs. It can be obtained from Eqs. (2.8)-(2.21) by replacing $W(\Gamma)$ in these equations by the Wilson loop of free electrodynamics [Eq. (3.6) with $\epsilon=1$ ]. Using Eq. (3.6) in (2.20) yields the classical electromagnetic field of the moving electron-positron pair and the resulting potential becomes one photon exchange. For example, this classical value of $\left\langle\left\langle F_{\mu \nu}(x)\right\rangle\right\rangle$ generates via Eqs. (2.12) and (2.13) the one photon exchange spin-orbit potential given by Eqs. (7.11) and (7.12) with $V_{0}(R)=-e^{2} /(4 \pi R)$ and $V_{2}^{\prime}(R)=e^{2} /\left(4 \pi R^{2}\right)=d V_{0}(R) / d R$.

In Yang-Mills theory, which is strongly coupled at large distances, $\left\langle\left\langle F_{\mu \nu}(x)\right\rangle\right\rangle$ does not take on its classical value and Eqs. (2.12) and (2.13) are not equivalent to one gluon exchange or indeed to the exchange of any single vector object. However its Lorentz transformation properties make evident the Lorentz vector nature of the potential. This is primarily a kinematic result that is a consequence of the following assumptions.

(1) The quark-antiquark interaction is described by QCD.

(2) Terms of order (quark mass) ${ }^{-2}$ are retained in the expansion of the $q \bar{q}$ Green's function in inverse powers of the quark mass. (Beyond this order radiation must be accounted for, and the potential is no longer defined.)

(3) Closed loops of quark pairs are omitted.

The dynamical manifestation of this vector coupling depends upon the behavior of $\left\langle\left\langle F_{\mu \nu}(x)\right\rangle\right\rangle$, which we determine in Secs. V and VII using the classical approximation to the dual theory. To clarify the technical details in these sections we describe some of them here. Equation (5.10) expresses $\left\langle\left\langle F_{\mu \nu}(x)\right\rangle\right\rangle$ as the dual of the corresponding tensor $G_{\mu \nu}$ of the dual theory and Eqs. (5.15) and (5.17) reexpress Eqs. (2.12) and (2.13) in terms of the color electric and magnetic components of this tensor denoted $\vec{D}$ and $\vec{H}$, respectively. Equation (5.15) is the usual expression for $V_{\mathrm{LS}}$ in terms of the color magnetic field in the comoving frame.

In Sec. VII we use the classical solution of the dual theory to evaluate this expression for $V_{\mathrm{LS}}$ at long distances. Equa- tion (7.2) expresses the color magnetic field in the comoving frame in terms of the classical solution $C_{\mu}$ of the equation of motion (6.2) of the dual theory. This equation differs from Eq. (3.17) (describing electrodynamics in the dual representation) by the presence of the monopole current that screens the color field of the quarks so that at large $R$ a color electric Abrikosov-Nielsen-Olesen vortex forms between the moving $q \bar{q}$ pair. This solution generates a spin-orbit potential determined by $V_{2}^{\prime}(R)$ (7.10) that for small $R$ approaches the one gluon exchange result and which for large $R$ decreases like $1 / R$.

Since the same classical solution generates a static potential that for large $R$ is linear in $R$, the spin-orbit potential cannot be described by vector exchange, for which $V_{2}^{\prime}(R)=d V_{0} / d R$ for all $R(6)$. This potential arises rather from the vector structure of Eq. (2.12) taken together with the classical solution of the dual theory, and differs from one gluon exchange because of the nonlinearity of the classical equations produced by the monopole current. At large quark separations where Yang-Mills theory becomes strongly coupled only the dual description is appropriate and the monopole current is essential. At small separations, on the other hand, the color field generated by the quarks expels the monopole condensate from the region between them, and the potential does become one gluon exchange.

Note that the specific result that $V_{2}^{\prime}(R)$ vanishes for large $R$, which follows from Eq. (7.10), obtains also in a model where the effective long distance $q \bar{q}$ interaction comes from scalar exchange, for which $V_{2}^{\prime}(R)=0$ for all $R(6)$. We have seen here that this long-distance limit of $V_{2}^{\prime}(R)$ is a consequence of the vector coupling of quarks in QCD combined with the dual treatment of long-distance Yang-Mills theory.

The essence of the constituent quark model is that the same potential can also be used to calculate the energy levels of mesons containing light quarks with constituent masses fixed by hadron spectroscopy. The assumption is that the principal effect of the light quark dynamics can be accounted for by giving the light quarks effective masses that become the parameters of the constituent quark model. The vector nature of $V_{q \bar{q}}$ is crucial to any relativistic treatment of the spin and kinematics of constituent quarks.

Finally we note the following "modified area law" proposed in Ref. [9]: $i \ln W(\Gamma)$ is written as the sum of a shortrange (SR) contribution and a long-range (LR) one:

$$
i \ln W(\Gamma)=i \ln W^{\mathrm{SR}}(\Gamma)+i \ln W^{\mathrm{LR}}(\Gamma),
$$

with $i \ln W^{\mathrm{SR}}(\Gamma)$ given by ordinary perturbation theory and

$$
i \ln W^{\mathrm{LR}}(\Gamma)=\sigma S_{\min },
$$

where $S_{\min }$ is the minimal surface enclosed by the loop $\Gamma$ and $\sigma$ is the string tension. We will show in Sec. VI that these two components of $i \ln W(\Gamma)$ arise as two limits of a single classical solution of the dual theory.

\section{THE DUAL DESCRIPTION OF ELECTRODYNAMICS}

Consider a pair of particles with charges $e(-e)$ moving along trajectories $\vec{z}_{1}(t)\left[\vec{z}_{2}(t)\right]$ in a relativistic medium hav- 
ing dielectric constant $\epsilon$. The current density $j^{\mu}(x)$ then has the form

$$
j^{\mu}(x)=e \oint_{\Gamma} d z^{\mu} \delta(x-z),
$$

where $\Gamma$ is the world line described in Fig. 1. In the usual $A_{\mu}$ (electric) description this system is described by a Lagrangian

$$
\mathcal{L}_{A}(j)=-\frac{\epsilon}{4}\left(\partial_{\alpha} A_{\beta}-\partial_{\beta} A_{\alpha}\right)^{2}-j^{\alpha} A_{\alpha},
$$

from which one obtains the usual Maxwell equations

$$
\partial^{\alpha} \epsilon\left(\partial_{\alpha} A_{\beta}-\partial_{\beta} A_{\alpha}\right)=j_{\beta} .
$$

If the (wave-number dependent) dielectric constant $\epsilon \rightarrow 0$ at long distances, then we see from Eq. (3.3) that $A_{\mu}$ is strongly coupled at long distances (antiscreening). From Eqs. (3.1) and (3.2) we have

$$
\int d x \mathcal{L}_{A}(j)=-\int d x \frac{\epsilon\left(\partial_{\mu} A_{\nu}-\partial_{\nu} A_{\mu}\right)^{2}}{4}-e \oint_{\Gamma} d z^{\mu} A_{\mu}(z) .
$$

The functional integral defining $W(\Gamma)$ in electrodynamics

$$
W(\Gamma)=\frac{\int \mathcal{D} A_{\mu} \exp \left(i \int d x\left[\mathcal{L}_{A}(j)+\mathcal{L}_{\mathrm{GF}}\right]\right)}{\int \mathcal{D} A_{\mu} \exp \left(i \int d x\left[\mathcal{L}_{A}(j=0)+\mathcal{L}_{\mathrm{GF}}\right]\right)},
$$

where $\mathcal{L}_{\mathrm{GF}}$ is a gauge fixing term, is Gaussian and has the value

$$
W(\Gamma)=\exp \left(-\frac{i e^{2}}{2} \oint_{\Gamma} d x^{\mu} \oint_{\Gamma} d x^{\prime \nu} \frac{D_{\mu \nu}\left(x-x^{\prime}\right)}{\epsilon}\right),
$$

where $D_{\mu \nu}$ is the free photon propagator and where selfenergies have been subtracted. Because of current conservation the result (3.6) is independent of the choice of gauge. Letting $\epsilon=1$ and expanding $i \ln W(\Gamma)$ to second order in the velocities, as in Eq. (2.4), gives the Darwin Lagrangian $L_{D}$ describing the interaction of a pair of oppositely charged particles $[13,14]$ :

$$
L_{D}=\frac{e^{2}}{4 \pi R}-\frac{1}{2} \frac{e^{2}}{4 \pi R}\left[\vec{v}_{1} \cdot \vec{v}_{2}+\frac{\left(\vec{v}_{1} \cdot \vec{R}\right)\left(\vec{v}_{2} \cdot \vec{R}\right)}{R^{2}}\right] .
$$

In the dual description we consider first the inhomogeneous Maxwell equations, which we write in the form

$$
-\partial^{\beta} \frac{\epsilon_{\alpha \beta \sigma \lambda} G^{\sigma \lambda}}{2}=j_{\alpha},
$$

where $G_{\mu \nu}$ is the dual field tensor composed of the electric displacement vector $\vec{D}$ and the magnetic field vector $\vec{H}$ :

$$
G_{0 k} \equiv H_{k}, \quad G_{\ell m}=\epsilon_{\ell m n} D^{n} .
$$

Next we express the charged particle current in Eq. (3.8) as the divergence of a polarization tensor $G_{\mu \nu}^{S}$ the Dirac string tensor, representing a moving line of polarization running from the negatively charged to the positively charged particle, namely, [15]:

$$
G_{\mu \nu}^{S}(x)=-e \epsilon_{\mu \nu \alpha \beta} \int d \sigma \int d \tau \frac{\partial y^{\alpha}}{\partial \sigma} \frac{\partial y^{\beta}}{\partial \tau} \delta[x-y(\sigma, \tau)],
$$

where $y^{\alpha}(\sigma, \tau)$ is a world sheet with boundary $\Gamma$ swept out by the Dirac string. Then [15]

$$
-\partial^{\beta} \frac{\epsilon_{\alpha \beta \sigma \lambda} G^{S \sigma \lambda}(x)}{2}=j_{\alpha}(x),
$$

and the solution of Eq. (3.8) is

$$
G_{\mu \nu}=\partial_{\mu} C_{\nu}-\partial_{\nu} C_{\mu}+G_{\mu \nu}^{S},
$$

which defines the magnetic variables (the dual potentials $C_{\mu}$ ). [With Eqs. (3.11) and (3.12) the inhomogeneous Maxwell equations become Bianchi identities.]

The homogeneous Maxwell equations for $\vec{E}$ and $\vec{B}$, which we write in the form

$$
\partial^{\alpha}\left(\mu G_{\alpha \beta}\right)=0,
$$

where $\mu=(1 / \epsilon)$ is the magnetic susceptibility, now become dynamical equations for the dual potentials. These equations can be obtained by varying $C_{\mu}$ in the Lagrangian

$$
\mathcal{L}_{C}\left(G_{\mu \nu}^{S}\right)=-\frac{1}{4} \mu G_{\mu \nu} G^{\mu \nu},
$$

where $G_{\mu \nu}$ is given by Eq. (3.12). This Lagrangian provides the dual (magnetic) description of the Maxwell theory (3.2). In the dual description the Wilson loop $W(\Gamma)$ is given by

$$
W(\Gamma) \equiv \frac{\int \mathcal{D} C_{\mu} \exp \left(i \int d x\left[\mathcal{L}_{C}\left(G_{\mu \nu}^{S}\right)+\mathcal{L}_{\mathrm{GF}}\right]\right)}{\int \mathcal{D} C_{\mu} \exp \left(i \int d x\left[\mathcal{L}_{C}\left(G_{\mu \nu}^{S}=0\right)+\mathcal{L}_{\mathrm{GF}}\right]\right)}
$$

Evaluating the functional integral (3.15) by completing the square gives

$$
W(\Gamma)=e^{-(i \mu / 4) \int d x G_{\alpha \beta}^{S}(x) G^{\alpha \beta}(x)},
$$

where $G^{\alpha \beta}(x)$ is the dual field tensor (3.12) with $C_{\mu}=C_{\mu}^{D}$ determined from the solution of Eq. (3.13), which has the explicit form

$$
\partial^{\alpha} \mu\left(\partial_{\alpha} C_{\beta}^{D}-\partial_{\beta} C_{\alpha}^{D}\right)=-\partial^{\alpha} \mu G_{\alpha \beta}^{S} .
$$

Solving Eq. (3.17), inserting the resulting expression for $G^{\alpha \beta}$ into Eq. (3.16), and using Eq. (3.11), we obtain the same result [Eq. (3.6) with $1 / \epsilon \rightarrow \mu$ ] for the Wilson loop (3.15) defined in the magnetic description as we had obtained for the Wilson loop defined in the electric description. We then have two equivalent descriptions at all distances of the electromagnetic interaction of two charged particles. [Note, however, that if $\epsilon \rightarrow 0$ at long distances, then $\mu \rightarrow 1 / \epsilon \rightarrow \infty$ and the dual potentials $C_{\mu}$ determined from Eq. (3.13) are not strongly coupled at long distances unlike the potentials $A_{\mu}$ determined from Eq. (3.3).] 


\section{THE DUAL DESCRIPTION OF LONG-DISTANCE YANG-MILLS THEORY}

The dual theory is an effective theory of long-distance Yang-Mills theory described by a Lagrangian density $\mathcal{L}_{\text {eff }}$ in which the fundamental variables are an octet of dual potentials $C_{\mu}$ coupled minimally to three octets of scalar Higgs fields $B_{i}$ carrying magnetic color charge. [The gauge coupling constant of dual theory $g=2 \pi / e$ where $e$ is the YangMills coupling constant.] The monopole fields $B_{i}$ develop nonvanishing vacuum expectation values $B_{0 i}$ (monopole condensation) that give rise to massive $C_{\mu}$ and consequently to a dual Meissner effect. Dual potentials couple to electric color charge like ordinary potentials couple to monopoles. The potentials $C_{\mu}$ thus couple to a quark-antiquark pair via a Dirac string connecting the pair. The dual Meissner effect prevents the electric color flux from spreading out as the distance $R$ between the quark-antiquark pair increases. As a result a linear potential develops that confines the quarks in hadrons. The dual theory then provides a concrete realization of the Mandelstam-'t Hooft [16] dual superconductor picture of confinement.

Because the quanta of the potentials $C_{\mu}$ are massive, the dual theory is weakly coupled at distances $R>1 / M$ ( $M$ being either the mass of the dual gluon or of the monopole field), and a semiclassical expansion can be used to calculate the heavy quark potential at those distances. The classical approximation gives the leading contribution to functional integrals defined by $\mathcal{L}_{\text {eff }}$, in contrast to the functional integrals of Yang-Mills theory where no single configuration of gauge potentials dominates $W(\Gamma)$. The duality assumption that the long-distance physics of Yang-Mills theory depending upon strongly coupled gauge potentials $A_{\mu}$ is the same as the longdistance physics of the dual theory describing the interactions of weakly coupled dual potentials $C_{\mu}$ and monopole fields $B_{i}$ forms the basis of the work of this paper.

In absence of sources $\mathcal{L}_{\text {eff }}$, has the form [1]

$$
\mathcal{L}_{\text {eff }}=2 \operatorname{tr}\left[-\frac{1}{4} \mathbf{G}^{\mu \nu} \mathbf{G}_{\mu \nu}+\frac{1}{2}\left(\mathcal{D}_{\mu} \mathbf{B}_{i}\right)^{2}\right]-W\left(\mathbf{B}_{i}\right),
$$

where

$$
\begin{gathered}
\mathcal{D}_{\mu} \mathbf{B}_{i}=\partial_{\mu} \mathbf{B}_{i}-i g\left[\mathbf{C}_{\mu}, \mathbf{B}_{i}\right], \\
\mathbf{G}_{\mu \nu}=\partial_{\mu} \mathbf{C}_{\nu}-\partial_{\nu} \mathbf{C}_{\mu}-i g\left[\mathbf{C}_{\mu}, \mathbf{C}_{\nu}\right], \\
g=\frac{2 \pi}{e}
\end{gathered}
$$

$\mathbf{C}_{\mu}$ and $\mathbf{B}_{i}$ are $\mathrm{SU}(3)$ matrices, and $W\left(\mathbf{B}_{i}\right)$ is the Higgs potential that has a minimum at nonzero values $\mathbf{B}_{0 i}$ that have the color structure

$$
\mathbf{B}_{01}=B_{0} \lambda_{7}, \quad \mathbf{B}_{02}=B_{0}\left(-\lambda_{5}\right), \quad \mathbf{B}_{03}=B_{0} \lambda_{2} .
$$

The three matrices $\lambda_{7},-\lambda_{5}$, and $\lambda_{2}$ transform as a $j=1$ irreducible representation of an $\mathrm{SU}(2)$ subgroup of $\mathrm{SU}(3)$ and as there is no $\mathrm{SU}(3)$ transformation that leaves all three $\mathbf{B}_{0 i}$ invariant the dual SU(3) gauge symmetry is completely broken and the eight Goldstone bosons become the longitudinal components of the now massive $\mathbf{C}_{\mu}$.
The basic manifestation of the dual superconducting properties of $\mathcal{L}_{\text {eff }}$ is that it generates classical equations of motion having solutions [17] carrying a unit of $Z_{3}$ flux confined in a narrow tube along the $z$ axis (corresponding to having quark sources at $z= \pm \infty$ ). (These solutions are dual to AbrikosovNielsen-Olesen magnetic vortex solutions [18] in a superconductor.) We briefly describe these classical solutions here in order to specify the color structures that enter into the subsequent treatment of the dual theory with quark sources that is not restricted to the classical approximation. We look for solutions where the dual potential is proportional to the hypercharge matrix $Y=\lambda_{8} / \sqrt{3}$ :

$$
\mathbf{C}_{\mu}=C_{\mu} Y
$$

As a consequence the non-Abelian terms in the expression (4.3) for the dual field tensor $\mathbf{G}_{\mu \nu}$ vanish.

We choose Higgs Fields $\mathbf{B}_{i}$ having the color structure

$$
\begin{aligned}
& \mathbf{B}_{1}=B_{1}(x) \lambda_{7}+\bar{B}_{1}(x)\left(-\lambda_{6}\right), \\
& \mathbf{B}_{2}=B_{2}(x)\left(-\lambda_{5}\right)+\bar{B}_{2}(x) \lambda_{4}, \\
& \mathbf{B}_{3}=B_{3}(x) \lambda_{2}+\bar{B}_{3}(x)\left(-\lambda_{1}\right) .
\end{aligned}
$$

With this ansatz the Higgs potential $W$ turns out to be

$$
\begin{aligned}
W= & \frac{2}{3} \lambda\left\{11\left[\left(\left|\phi_{1}\right|^{2}-B_{0}^{2}\right)^{2}+\left(\left|\phi_{2}\right|^{2}-B_{0}^{2}\right)^{2}+\left(\left|\phi_{3}\right|^{2}-B_{0}^{2}\right)^{2}\right]\right. \\
& \left.+7\left(\left|\phi_{1}\right|^{2}+\left|\phi_{2}\right|^{2}+\left|\phi_{3}\right|^{2}-3 B_{0}^{2}\right)^{2}\right\}
\end{aligned}
$$

where

$$
\phi_{i}(x)=B_{i}(x)-i \bar{B}_{i}(x)
$$

Using Eqs. (4.5) and (4.6) we also find

$$
\begin{aligned}
2 \operatorname{tr} \sum_{i}\left(\mathcal{D}_{\mu} \mathbf{B}_{i}\right)^{2}= & 4\left|\left(\partial_{\mu}-i g C_{\mu}\right) \phi_{1}\right|^{2}+4\left|\left(\partial_{\mu}-i g C_{\mu}\right) \phi_{2}\right|^{2} \\
& +4\left|\partial_{\mu} \phi_{3}\right|^{2}
\end{aligned}
$$

Since $\phi_{1}$ and $\phi_{2}$ couple to $C_{\mu}$ in the same way and $\phi_{3}$ does not couple to $C_{\mu}$ at all, we can choose $\phi_{1}=\phi_{2}=\phi \equiv B-i \bar{B}$, and $\phi_{3}=B_{3}$, so that

$$
2 \operatorname{tr} \sum_{i}\left(\mathcal{D}_{\mu} \mathbf{B}_{i}\right)^{2}=8\left|\left(\partial_{\mu}-i g C_{\mu}\right) \phi\right|^{2}+4\left(\partial_{\mu} B_{3}\right)^{2}
$$

At large distances from the center of the flux tube, using cylindrical coordinates $\rho, \theta, z$ we have the boundary conditions

$$
\vec{C} \rightarrow-\frac{\hat{e}_{\theta}}{g \rho}, \quad \phi \rightarrow B_{0} e^{i \theta}, \quad B_{3} \rightarrow B_{0}, \quad \text { as } \rho \rightarrow \infty
$$

The nonvanishing of $B_{0}$ produces a color monopole current confining the electric color flux. The line integral of the dual potential around a large loop surrounding the $z$ axis measures the electric color flux, just as the corresponding line integral 
of the ordinary vector potential measures the magnetic flux in a superconducting vortex. Since the dual potential is along a single direction in color space path ordering is unnecessary and the boundary condition (4.11) for $\overrightarrow{\mathbf{C}}$ gives

$$
e^{-i g \oint_{\text {loop }} \overrightarrow{\mathbf{C}} \cdot d \vec{\ell}}=e^{2 \pi i Y}=e^{2 \pi(i / 3)},
$$

which manifests the unit of $Z_{3}$ flux in the tube. [A continuous deformation in $\mathrm{SU}(3)$ of our particular solution into a non-Abelian configuration will leave unchanged the path ordered integral $\left.P \exp \left(-i g \int \overrightarrow{\mathbf{C}} \cdot d \vec{\ell}\right)=e^{2 \pi i / 3}\right)$. The energy per unit length in this flux tube is the string tension $\sigma$. The quantity $g^{2} / \lambda$ plays the role of a Landau-Ginzburg parameter. Its value can be obtained by relating the difference between the energy density at a large distance from the flux tube and the energy density at its center to the gluon condensate. This procedure gives $g^{2} / \lambda=5$. We get from the numerical integration of the static field equations [17]

$$
\sigma \approx 1.1\left(24 B_{0}^{2}\right)
$$

We are left with two free parameters in $\mathcal{L}_{\text {eff }}$, which we take to be $\alpha_{s}=e^{2} / 4 \pi=\pi / g^{2}$ and the string tension $\sigma$.

To couple $\mathbf{C}_{\mu}$ to a $q \bar{q}$ pair separated by a finite distance we must represent quark sources by a Dirac string tensor $\mathbf{G}_{\mu \nu}^{S}$ We choose the dual potential to have the same color structure (4.5) as the flux tube solution. Then $\mathbf{G}_{\mu \nu}^{S}$ must also be proportional to the hypercharge matrix

$$
\mathbf{G}_{\mu \nu}^{S}=Y G_{\mu \nu}^{S},
$$

where $G_{\mu \nu}^{S}$ is given by Eq. (3.10), so that one unit of $Z_{3}$ flux flows along the Dirac string connecting the quark and antiquark. We then couple quarks by replacing $\mathbf{G}_{\mu \nu}$ in $\mathcal{L}_{\text {eff }}(4.1)$ by

$$
\mathbf{G}_{\mu \nu}=Y G_{\mu \nu},
$$

where

$$
G_{\mu \nu}=\partial_{\mu} C_{\nu}-\partial_{\nu} C_{\mu}+G_{\mu \nu}^{S}
$$

Inserting Eq. (4.15) into Eq. (4.1) and using Eq. (4.10) then yields the Lagrangian $\mathcal{L}_{\text {eff }}\left(G_{\mu \nu}^{S}\right)$ coupling dual potentials to classical quark sources moving along trajectories $\vec{z}_{1}(t)$ and $\vec{z}_{2}(t)$ :

$$
\begin{aligned}
\mathcal{L}_{\mathrm{eff}}\left(G_{\mu \nu}^{S}\right)= & -\frac{4}{3} \frac{\left(\partial_{\mu} C_{\nu}-\partial_{\nu} C_{\mu}+G_{\mu \nu}^{S}\right)^{2}}{4} \\
& +\frac{8\left|\left(\partial_{\mu}-i g C_{\mu}\right) \phi\right|^{2}}{2}+\frac{4\left(\partial_{\mu} B_{3}\right)^{2}}{2}-W,
\end{aligned}
$$

where $W$ is given by Eq. (4.7) with $\phi_{1}=\phi_{2}=\phi, \phi_{3}=B_{3}$.

It is useful, as in Eq. (3.9), to decompose $G_{\mu \nu}$ into its color electric components $\vec{D}$ and color magnetic components $\vec{H}$. Similarly we decompose $G_{\mu \nu}^{S}$ into its polarization components $\vec{D}_{S}$ and its magnetization components $\vec{H}_{S}$ :

$$
D_{S}^{k}=\frac{1}{2} \epsilon_{k m n} G^{S m n}, \quad H_{S}^{k}=G_{0 k}^{S} .
$$

Then Eq. (4.16) becomes

$$
\vec{D}=-\vec{\nabla} \times \vec{C}+\vec{D}_{S}, \quad \vec{H}=-\vec{\nabla} C_{0}-\frac{\partial \vec{C}}{\partial t}+\vec{H}_{S}
$$

The Lagrangian density $\mathcal{L}_{\text {eff }}\left(G_{\mu \nu}^{S}\right)$ (4.17) can then be written as the sum of an "electric" part $\mathcal{L}_{0}$ and a "magnetic" part $\mathcal{L}_{2}$ : i.e.,

$$
\mathcal{L}_{\text {eff }}\left(G_{\mu \nu}^{S}\right)=\mathcal{L}_{0}+\mathcal{L}_{2}
$$

where

$$
\mathcal{L}_{0}=-\left\{\frac{2}{3} \vec{D}^{2}+4|(\vec{\nabla}+i g \vec{C}) \phi|^{2}+2\left(\vec{\nabla} B_{3}\right)^{2}+W\right\},
$$

and

$$
\mathcal{L}_{2}=\frac{2}{3} \vec{H}^{2}+4\left|\left(\partial_{0}-i g C_{0}\right) \phi\right|^{2}+2\left(\partial_{0} B_{3}\right)^{2}
$$

and all terms involving time derivatives appear only in $\mathcal{L}_{2}$.

We denote by $W_{\text {eff }}(\Gamma)$ the Wilson loop of the dual theory: i.e.,

$W_{\mathrm{eff}}(\Gamma)=\frac{\int \mathcal{D} C_{\mu} \mathcal{D} \phi \mathcal{D} B_{3} \exp \left(i \int d x\left[\mathcal{L}_{\mathrm{eff}}\left(G_{\mu \nu}^{S}\right)+\mathcal{L}_{\mathrm{GF}}\right]\right)}{\int \mathcal{D} C_{\mu} \mathcal{D} \phi \mathcal{D} B_{3} \exp \left(i \int d x\left[\mathcal{L}_{\mathrm{eff}}\left(G_{\mu \nu}^{S}=0\right)+\mathcal{L}_{\mathrm{GF}}\right]\right)}$.

The functional integral $W_{\text {eff }}(\Gamma)$ Eq. (4.23) determines in the dual theory the same physical quantity as $W(\Gamma)$ in YangMills theory, namely the action for a quark-antiquark pair moving along classical trajectories. The coupling in $\mathcal{L}_{\text {eff }}\left(G_{\mu \nu}^{S}\right)$ of dual potentials to Dirac strings plays the role in the expression (4.23) for $W_{\text {eff }}(\Gamma)$ of the explicit Wilson loop integral $e^{-i e \oint_{\Gamma} d x^{\mu} A_{\mu}(x)}$ in the expression (2.2) for $^{2} W(\Gamma)$.

The assumption that the dual theory describes the longdistance $q \bar{q}$ interaction in Yang-Mills theory then takes the form

$$
W(\Gamma)=W_{\mathrm{eff}}(\Gamma), \quad \text { for large loops } \Gamma \text {. }
$$

Large loops means that the size $R$ of the loop is large compared to the inverse mass of the Higgs particle (monopole field) $\phi$. Furthermore since the dual theory is weakly coupled at large distances we can evaluate $W_{\text {eff }}(\Gamma)$ via a semiclassical expansion to which the classical configuration of dual potentials and monopoles gives the leading contribution. This then allows us to picture heavy quarks (or constituent quarks) as sources of a long-distance classical field of dual gluons determining the heavy quark potential. Thus, in a

\footnotetext{
${ }^{2}$ We emphasize the distinction between $W_{\text {eff }}(\Gamma)$ and the dual Wilson loop defined as an average of $e^{i g \phi \vec{C} \cdot d \vec{\ell}}$. This dual Wilson loop would describe the interaction of a monopole-antimonopole pair. For large loops the dual Wilson loop satisfies a perimeter law.
} 
certain sense the dual gluon fields $G_{\mu \nu}$ mediate the heavy quark interaction just as the electromagnetic field mediates the electron-positron interaction.

Using the duality hypothesis, we replace $W(\Gamma)$ by $W_{\text {eff }}(\Gamma)$ in Eqs. (2.4)-(2.6) to obtain expressions for $V_{0}(R)$ and $V_{\mathrm{VD}}$ in the dual theory as the zero-order and quadratic terms in the expansion of $i \ln W_{\text {eff }}(\Gamma)$ for small velocities $\vec{z}_{1}$ and $\vec{z}_{2}$, i.e., the interaction Lagrangian $L_{I}$, calculated in the dual theory, is obtained from the equation

$$
i \ln W_{\mathrm{eff}}(\Gamma)=-\int_{t_{i}}^{t_{t}} d t L_{I}\left(\vec{z}_{1}, \vec{z}_{2}, \vec{z}_{1}, \vec{z}_{2}\right) .
$$

\section{Remark}

There has been a recent revival of interest in the role of electric-magnetic duality due to the work of Seiberg and Witten [7] and Seiberg [8] on supersymmetric non-Abelian gauge theories. Seiberg considered $\mathrm{SU}\left(N_{c}\right)$ gauge theory with $N_{f}$ flavors of massless quarks. Although he did not exhibit an explicit duality transformation he inferred the complete structure of the magnetic gauge group and hence the associated massless particle content of the dual Lagrangian. For a certain range of $N_{f}$ the dual theory is weakly coupled at large distances and hence the low-energy spectrum of the theory consists just of the massless particles of the dual Lagrangian. Since this dual "magnetic" Lagrangian describes the same low-energy physics as the original Lagrangian, the particle spectrum, mirroring the magnetic gauge group, must appear as composites of the original "electric" gauge degrees of freedom. For $N_{f}=N_{c}+1$ the dual gauge group is completely broken, the associated dual gauge bosons become massive and the quarks of the original theory are confined.

There are obvious differences between Seiberg's example where the number of massless fermions plays an essential role, and the example of Yang-Mills theory where neither the original theory nor the proposed dual Lagrangian $\mathcal{L}_{\text {eff }}$ contains fermions. Here confinement manifests itself via the development of a linear potential between heavy quark sources, whereas in the supersymmetric models confinement manifests itself via the realization of the hadron spectrum as composites of the original quark variables. In the supersymmetric model these hadrons are massless and as usual the production of these particles prevents the development of a linear potential. However, all the gauge bosons of the dual theory are massive and the coupling of the pure gauge sector to quark sources would produce a long-distance linear potential between these sources. The common feature of Seiberg's supersymmetric model, where duality is "inferred," and YangMills theory, where duality is conjectured, is that in both cases the dual gluons receive mass via a Higgs mechanism, which is the essential element of the dual superconductor mechanism.

\section{THE POTENTIAL $V_{q \bar{q}}$ IN THE DUAL THEORY}

We now express the spin-dependent heavy quark potential $V_{\mathrm{SD}}(2.8)$ in terms of quantities of the dual theory. As a first step we find relations of matrix elements of the dual field tensor $G_{\mu \nu}$ to variations of $W_{\text {eff }}(\Gamma)$ that are analogous to Eq. (2.20) relating $\left\langle\left\langle F_{\mu \nu}\right\rangle\right\rangle$ to variations in $W(\Gamma)$. Consider the variation in $W_{\text {eff }}(\Gamma)$ produced by the change

$$
G_{\mu \nu}^{S}(x) \rightarrow G_{\mu \nu}^{S}(x)+\delta G_{\mu \nu}^{S}(x) .
$$

From Eq. (4.23) we find that the corresponding variation $\delta W_{\text {eff }}(\Gamma)$ is given by

$$
\delta i \ln W_{\mathrm{eff}}(\Gamma)=\frac{4}{3} \int d x \frac{\delta G_{\mu \nu}^{S}(x)}{2}\left\langle\left\langle G^{\mu \nu}(x)\right\rangle\right\rangle_{\mathrm{eff}},
$$

where

$$
\left\langle\left\langle f\left(C_{\mu}, \phi, B_{3}\right)\right\rangle\right\rangle_{\mathrm{eff}} \equiv \frac{\int \mathcal{D} C_{\mu} \mathcal{D} \phi \mathcal{D} B_{3} \exp \left(i \int d x\left[\mathcal{L}_{\mathrm{eff}}\left(G_{\mu \nu}^{S}\right)+\mathcal{L}_{\mathrm{GF}}\right]\right) f\left(C_{\mu}, \phi, B_{3}\right)}{\int \mathcal{D} C_{\mu} \mathcal{D} \phi \mathcal{D} B_{3} \exp \left(i \int d x\left[\mathcal{L}_{\mathrm{eff}}\left(G_{\mu \nu}^{S}\right)+\mathcal{L}_{\mathrm{GF}}\right]\right)}
$$

Using Eq. (3.10) to express the variation of $G_{\mu \nu}^{S}$ in terms of the variation of the world sheet $y^{\mu}(\sigma, \tau)$, we obtain

$$
\begin{aligned}
\int d x \frac{\delta G_{\mu \nu}^{S}(x)}{2} & \left\langle\left\langle G^{\mu \nu}(x)\right\rangle\right\rangle_{\mathrm{eff}} \\
= & -\frac{e}{2} \epsilon_{\mu \nu \lambda \alpha} \int_{t_{i}}^{t_{f}} d \tau\left[\delta z_{1}^{\alpha} \frac{\partial z_{1}^{\lambda}}{\partial \tau}\left\langle\left\langle G^{\mu \nu}\left(z_{1}\right)\right\rangle\right\rangle_{\mathrm{eff}}\right. \\
& \left.-\delta z_{2}^{\alpha} \frac{\partial z_{2}^{\lambda}}{\partial \tau}\left\langle\left\langle G^{\mu \nu}\left(z_{2}\right)\right\rangle\right\rangle_{\mathrm{eff}}\right] .
\end{aligned}
$$

The right-hand side of Eq. (5.4) arises from varying the boundary of the Dirac sheet. The variation of the interior of the sheet produces a contribution proportional to the monopole current $j_{\nu}^{\text {mon }}$

$$
j_{\nu}^{\mathrm{mon}}(x) \equiv \partial^{\mu} G_{\mu \nu}(x)
$$

[See Eq. (A52) of Ref. [1] for details.] This gives no additional contribution to Eq. (5.4) since the monopole current must vanish on the Dirac sheet, so that no monopole can pass through the Dirac string connecting the charged particles. 
This latter assertion is just the dual of Dirac's condition for the consistency of a theory containing both electric charges and monopoles [15].

Defining $d z^{\lambda} \equiv d \tau \partial z^{\lambda} / \partial \tau$, we can then write Eq. (5.4) as

$$
\begin{aligned}
\int & d x \frac{\delta G_{\mu \nu}^{S}(x)}{2}\left\langle\left\langle G^{\mu \nu}(x)\right\rangle\right\rangle_{\mathrm{eff}} \\
& =-e \int\left(\delta z_{1}^{\alpha} d z_{1}^{\lambda}\left\langle\left\langle\hat{G}_{\lambda \alpha}\left(z_{1}\right)\right\rangle\right\rangle_{\mathrm{eff}}\right. \\
& \left.-\delta z_{2}^{\alpha} d z_{2}^{\lambda}\left\langle\left\langle\hat{G}_{\lambda \alpha}\left(z_{2}\right)\right\rangle\right\rangle_{\mathrm{eff}}\right),
\end{aligned}
$$

where

$$
\hat{G}_{\mu \nu}(x) \equiv \frac{1}{2} \epsilon_{\mu \nu \lambda \sigma} G^{\lambda \sigma}(x) .
$$

Choosing a variation that vanishes on the curve $\Gamma_{2}$, we obtain

$$
\delta i \ln W_{\mathrm{eff}}(\Gamma)=-\frac{4}{3} e \int \frac{\delta S^{\mu \nu}\left(z_{1}\right)}{2}\left\langle\left\langle\hat{G}_{\mu \nu}\left(z_{1}\right)\right\rangle\right\rangle_{\mathrm{eff}},
$$

where $\delta S^{\mu \nu}\left(z_{1}\right)$ is given by Eq. (2.19). Equations (5.2) and (5.8) can be written as

$$
\begin{aligned}
\frac{\delta i \ln W_{\mathrm{eff}}(\Gamma)}{\delta S^{\mu \nu}\left(z_{1}\right)} & =-\frac{4}{3} e\left\langle\left\langle\hat{G}_{\mu \nu}\left(z_{1}\right)\right\rangle\right\rangle_{\mathrm{eff}} \\
& =-\frac{e}{2} \varepsilon_{\mu \nu \lambda \sigma} \frac{\delta i \ln W_{\mathrm{eff}}(\Gamma)}{\delta G_{\lambda \sigma}^{S}\left(z_{1}\right)},
\end{aligned}
$$

which is the dual theory analogue of Eq. (2.20). The duality assumption (4.24) then gives a corresponding relation between matrix elements:

$$
\left\langle\left\langle F_{\mu \nu}\left(z_{1}\right)\right\rangle\right\rangle=\frac{4}{3}\left\langle\left\langle\hat{G}_{\mu \nu}\left(z_{1}\right)\right\rangle\right\rangle_{\mathrm{eff}} .
$$

Equation (5.10) gives a correspondence between local quantities in Yang-Mills theory and in the dual theory. The utility of electric-magnetic duality is that for large loops semiclassical configurations dominate the right-hand side of Eq. (5.10) in contrast to the rapidly fluctuating configurations of Yang-Mills potential that contribute to the left-hand side. Equation (5.10) breaks up into its electric and magnetic components

$$
\begin{gathered}
-\left\langle\left\langle F_{m n}\right\rangle\right\rangle=\frac{4}{3} \epsilon_{m n} \lambda\left\langle\left\langle H^{\ell}\right\rangle\right\rangle_{\mathrm{eff}}, \\
\left\langle\left\langle F_{0}\right\rangle\right\rangle=\frac{4}{3}\left\langle\left\langle D_{\ell}\right\rangle\right\rangle_{\mathrm{eff}},
\end{gathered}
$$

or, equivalently,

$$
\begin{gathered}
\left\langle\left\langle\hat{F}_{0} \ell\right\rangle=\frac{4}{3}\left\langle\left\langle H_{\ell}\right\rangle\right\rangle_{\mathrm{eff}},\right. \\
\left\langle\left\langle\hat{F}_{m n}\right\rangle\right\rangle=\frac{4}{3} \epsilon_{m n k}\left\langle\left\langle D_{k}\right\rangle\right\rangle_{\mathrm{eff}} .
\end{gathered}
$$

Using Eqs. (5.13) and (5.14) in Eq. (2.12) gives the following expression for $V_{\mathrm{LS}}^{\mathrm{mag}}$ in the dual theory:

$$
V_{\mathrm{LS}}^{\mathrm{mag}}=-\sum_{j=1}^{2} \frac{4}{3} \frac{e_{j}}{m_{j}} \vec{S}_{j} \cdot\left(\left\langle\left\langle\vec{H}\left(z_{j}\right)\right\rangle\right\rangle_{\mathrm{eff}}-\vec{v}_{j} \times\left\langle\left\langle\vec{D}\left(z_{j}\right)\right\rangle\right\rangle_{\mathrm{eff}}\right),
$$

where $e_{1}=e$ and $e_{2}=-e$. Note that $\langle\langle\vec{H}\rangle\rangle_{\mathrm{eff}}-\vec{v}_{j} \times\langle\langle\vec{D}\rangle\rangle_{\mathrm{eff}}$ is the color magnetic field at the $j$ th quark in the comoving Lorentz frame, $V_{\mathrm{LS}}^{\mathrm{mag}}$ the magnetic interaction of this field with a quark having a $g$ factor 2 . The fact that heavy quarks interact with a Dirac magnetic moment is a consequence of the $(1 / m)$ expansion [2] for the $q \bar{q}$ Green's function upon which this analysis is based.

To evaluate $V_{\text {Thomas }}(2.13)$ we note from Eq. (5.12) that

$$
\begin{aligned}
& \frac{e}{2 m_{1}^{2}} \int d z_{1}^{\mu} S_{1}^{\ell} \epsilon^{\ell k r} p_{1}^{k}\left\langle\left\langle F_{\mu r}\left(z_{1}\right)\right\rangle\right\rangle \\
& \quad=\frac{4}{3} \frac{e}{2 m_{1}^{2}} \int d t \vec{S}_{1} \cdot \vec{p}_{1} \times\left\langle\left\langle\vec{D}\left(z_{1}\right)\right\rangle\right\rangle_{\mathrm{eff}},
\end{aligned}
$$

and obtain

$$
V_{\text {Thomas }}=-\frac{1}{2} \sum_{j=1}^{2} \frac{4}{3} \frac{e_{j}}{m_{j}} S_{j} \cdot\left(\vec{v}_{j} \times\left\langle\left\langle\vec{D}\left(z_{j}\right)\right\rangle\right\rangle_{\mathrm{eff}}\right) .
$$

The expression (5.17) is the contribution to the potential due to the precession of the axis of the comoving frame. In Appendix A it is shown that Eq. (5.17) can be written in the usual form

$$
V_{\text {Thomas }}=\frac{1}{2 m_{1}} \frac{1}{R} \frac{d V_{0}}{d R} \vec{S}_{1} \cdot \vec{v}_{1} \times \vec{R}-\frac{1}{2 m_{2}} \frac{d V_{0}}{d R} \vec{S}_{2} \cdot \vec{v}_{2} \times \vec{R} .
$$

Equation (5.18) is essentially a kinematic relation and is independent of the dynamics of Yang-Mills theory. On the other hand $V_{\mathrm{LS}}^{\mathrm{mag}}$ (5.15) depends upon the dynamics and cannot be expressed solely in terms of the static potential.

To express $V_{\mathrm{SS}}$ (2.17) in terms of quantities involving the dual theory we need the following:

$$
\begin{aligned}
i e^{2} & \left\{\left\langle\left\langle\hat{F}_{/ 0}\left(z_{j}\right) \hat{F}_{k 0}\left(z_{j^{\prime}}^{\prime}\right)\right\rangle\right\rangle-\left\langle\left\langle\hat{F}_{\ell 0}\left(z_{j}\right)\right\rangle\right\rangle\left\langle\left\langle\hat{F}_{k 0}\left(z_{j^{\prime}}^{\prime}\right)\right\rangle\right\rangle\right\} \\
& =\frac{4}{3} e^{2} \frac{\delta\left\langle\left\langle H_{k}\left(z_{j}\right)\right\rangle\right\rangle_{\mathrm{eff}}}{\delta H_{S}\left(z_{j^{\prime}}^{\prime}\right)} .
\end{aligned}
$$

To obtain Eq. (5.19) we use Eqs. (2.21) and (5.13) and the equation

$$
\frac{\delta\left\langle\left\langle H_{k}\left(z_{j}\right)\right\rangle\right\rangle_{\mathrm{eff}}}{\delta G_{0 /}^{S}\left(z_{j^{\prime}}\right)}=-\frac{\epsilon_{\ell m n}}{2} \frac{\delta\left\langle\left\langle H_{k}\left(z_{j}\right)\right\rangle\right\rangle_{\mathrm{eff}}}{\delta S^{m n}\left(z_{j^{\prime}}\right)} .
$$

[Compare Eq. (5.9).] Using Eq. (5.19) in (2.17), we obtain

$$
\begin{aligned}
\int d t V_{\mathrm{SS}}= & -\frac{1}{2} \sum_{j, j^{\prime}=1}^{2} \frac{e^{2}}{m_{j} m_{j^{\prime}}} T_{s} \int_{\Gamma_{j}} d t \int_{\Gamma_{j^{\prime}}} d t^{\prime} S_{j}^{\prime} S_{j^{\prime}}^{k} \\
& \times\left(\frac{4}{3} \frac{\delta\left\langle\left\langle H_{k}\left(z_{j}\right)\right\rangle\right\rangle_{\mathrm{eff}}}{\delta H_{S}\left(z_{j^{\prime}}^{\prime}\right)}\right) .
\end{aligned}
$$


The factor multiplying $S_{j}^{\ell} S_{j^{\prime}}^{k}$ is symmetric in $k$ and $\ell$ and hence the terms in Eq. (5.21) where $j=j^{\prime}$ involve the combination

$$
\frac{S_{j}^{\ell} S_{j}^{k}+S_{j}^{k} S_{j}^{\ell}}{2}=\frac{1}{4} \delta_{k \ell}
$$

Equation (5.21) then becomes

$$
\begin{aligned}
\int d t V_{\mathrm{SS}}= & -\frac{4}{3} \sum_{j=1}^{2} \frac{e^{2}}{8 m_{j}^{2}} \int_{\Gamma_{j}} d t \int_{\Gamma_{j}} d t^{\prime} \frac{\delta\left\langle\left\langle H_{k}\left(z_{j}\right)\right\rangle\right\rangle_{\mathrm{eff}}}{\delta H_{S k}\left(z_{j}^{\prime}\right)} \\
& -\frac{4}{3}\left(\frac{e^{2}}{m_{1} m_{2}}\right) \int_{\Gamma_{1}} d t \int_{\Gamma_{2}} d t^{\prime} S_{1}^{k} S_{2}^{\ell} \frac{\delta\left\langle\left\langle H_{k}\left(z_{1}\right)\right\rangle\right\rangle_{\mathrm{eff}}}{\delta H_{S} \ell\left(z_{2}^{\prime}\right)} .
\end{aligned}
$$

The first term in Eq. (5.22) is a spin-independent velocityindependent contribution to the potential proportional to inverse square of the quark masses while the second term in Eq. (5.22) yields a spin-spin interaction of the expected structure.

Finally consider $V_{\text {Darwin }}(2.15)$ and note that

$$
\left\langle\left\langle D^{\nu} F_{\nu \mu}\left(z_{j}\right)\right\rangle\right\rangle=\partial^{\nu}\left\langle\left\langle F_{\nu \mu}\left(z_{j}\right)\right\rangle\right\rangle .
$$

The derivative of the Wilson loop occurring in the definition (2.10) of $\left\langle\left\langle F_{\nu \mu}(x)\right\rangle\right\rangle$ yields the Yang-Mills potential $A_{\nu}$ appearing in $D^{\nu} F_{\nu \mu}$. Using Eq. (5.10) we obtain ${ }^{3}$

$$
\int V_{\text {Darwin }} d t=-\frac{4}{3} \sum_{j} \frac{e}{8 m_{j}^{2}} \int_{\Gamma_{j}} d x^{\mu} \partial^{\nu}\left\langle\left\langle\hat{G}_{\nu \mu}(x)\right\rangle\right\rangle_{\mathrm{eff}} .
$$

For an alternative expression for $V_{\text {Darwin }}$ based on Eq. (A7) of Ref. [4] see Eq. (B3) of Appendix B.

\section{THE CLASSICAL APPROXIMATION FOR $V_{0}(R)$ AND $V_{V D}$}

In the classical approximation Eq. (4.23) becomes

$$
i \ln W_{\mathrm{eff}}=-\int d x \mathcal{L}_{\mathrm{eff}}\left(G_{\mu \nu}^{S}\right)
$$

where $\mathcal{L}_{\text {eff }}\left(G_{\mu \nu}^{S}\right)(4.17)$ is evaluated at the solution of the classical equations of motion:

\footnotetext{
${ }^{3}$ Notice that $\left\langle\left\langle F_{\mu \nu}(z)\right\rangle\right\rangle$ depends not only on the point $z$ but on the entire Wilson loop. So in order for Eq. (5.23) to make sense one has to use the appropriate definition of derivative. Given a functional $\Phi_{\left[\gamma_{a b}\right]}$ of the curve $\gamma_{a b}$ with ends $a$ and $b$, under general regularity condition the variation of $\Phi$ consequent to an infinitesimal modification of the curve $\gamma \rightarrow \gamma+\delta \gamma$ can be expressed as the sum of various terms proportional respectively to $\delta a, \delta b$ and to the elements $\delta S_{\rho \sigma}(x)$ of the surface swept by the curve. Then the derivatives $\partial / \partial a^{\rho}, \partial / \partial b^{\rho}$ and $\delta / \delta S^{\rho \sigma}(x)$ are defined by the equation $\delta \Phi=\partial \Phi / \partial a^{\rho} \delta a^{\rho}+\partial \Phi / \partial b^{\rho} \delta b^{\rho}+\int_{\gamma} \delta S^{\rho \sigma}(x) \delta \Phi / \delta S^{\rho \sigma}(x)$. In our case this would amount to put naively $\partial / \partial z^{\rho} P f\left(\int_{z}^{b} d x^{\mu} A_{\mu}(x)\right)=-P f^{\prime}\left(\int_{z}^{b} d x^{\mu} A_{\mu}(x)\right) A_{\rho}(z) \quad$ and $\partial / \partial z^{\rho} \int_{a}^{z} d x^{\mu} A_{\mu}(x)=A_{\rho}(z) P f^{\prime}\left(\int_{a}^{z} d x^{\mu} A_{\mu}(x)\right)$.
}

$$
\begin{gathered}
\partial^{\alpha}\left(\partial_{\alpha} C_{\beta}-\partial_{\beta} C_{\alpha}\right)=-\partial^{\alpha} G_{\alpha \beta}^{S}+j_{\beta}^{\text {mon }}, \\
\left(\partial_{\mu}-i g C_{\mu}\right)^{2} \phi=-\frac{1}{4} \frac{\delta W}{\delta \phi^{*}},
\end{gathered}
$$

and

$$
\partial^{2} B_{3}=-\frac{1}{4} \frac{\delta W}{\delta B_{3}}
$$

where the monopole current $j_{\mu}^{\text {mon }}$ is

$$
j_{\mu}^{\mathrm{mon}}=-3 i g\left[\phi^{*}\left(\partial_{\mu}-i g C_{\mu}\right) \phi-\phi\left(\partial_{\mu}+i g C_{\mu}\right) \phi^{*}\right] .
$$

As a result of the classical approximation all quantities in brackets are replaced by their classical values

$$
\left\langle\left\langle G_{\mu \nu}(x)\right\rangle\right\rangle_{\mathrm{eff}}=G_{\mu \nu}(x) .
$$

The electric and magnetic components of Eq. (6.6) are

$$
\langle\langle\vec{D}(x)\rangle\rangle_{\mathrm{eff}}=\vec{D}(\vec{x}), \quad\langle\langle\vec{H}(x)\rangle\rangle_{\mathrm{eff}}=\vec{H}(\vec{x}),
$$

where $\vec{D}$ and $\vec{H}$ are the color electric and magnetic fields, respectively, given in terms of the dual potentials by Eq. (4.19).

We choose the Dirac string to be a straight line $L$ connecting the quarks. As $\vec{x}$ approaches the string, $\phi(x) \rightarrow 0, C_{\mu}(x) \rightarrow C_{\mu}^{D}(x)$, satisfying Eq. (3.17). As $\vec{x} \rightarrow \infty, \quad \phi(x) \rightarrow B_{0}, \quad C_{\mu}(x) \rightarrow 0$, in contrast with the large distance boundary condition for the infinite flux tube. We can then choose $\phi(x)$ to be real so that

$$
\phi(x)=B(x), \quad j_{\mu}^{\mathrm{mon}}(x)=-6 g^{2} C_{\mu} B^{2} .
$$

Consider first the case of static quarks, $\vec{v}_{1}=\vec{v}_{2}=0$. Then the scalar potential $C_{0}$ and the color magnetic field $\vec{H}$ vanish, and $\mathcal{L}_{\text {eff }}$ reduces to $\mathcal{L}_{0}$ Eq. (4.21) that yields the static potential

$$
V_{0}(R)=-\int d \vec{x} \mathcal{L}_{0}
$$

where $\mathcal{L}_{0}$ is evaluated at the static solution of Eqs. (6.2)(6.4), which have the following form in this case:

$$
\begin{gathered}
-\vec{\nabla} \times(\vec{\nabla} \times \vec{C})-6 g^{2} B^{2} \vec{C}=-\vec{\nabla} \times \vec{D}_{S} \\
\left(-\nabla^{2}+g^{2} \vec{C}^{2}\right) B=-\frac{2 \lambda}{3} B\left(25 B^{2}+7 B_{3}^{2}-32 B_{0}^{2}\right),
\end{gathered}
$$

and

$$
-\nabla^{2} B_{3}=-\frac{4 \lambda}{3} B_{3}\left(7 B^{2}+9 B_{3}^{2}-16 B_{0}^{2}\right),
$$

where we have used the explicit form, Eq. (4.7), of W.

To solve Eq. (5.34) it is convenient to write

$$
\vec{C}=\vec{C}^{D}+\vec{c}
$$


where $\vec{C}^{D}$ is the Dirac potential satisfying the static form of Eq. (3.17): namely,

$$
-\vec{\nabla} \times\left(\vec{\nabla} \times \vec{C}^{D}\right)=-\vec{\nabla} \times \vec{D}_{S},
$$

with $\vec{D}_{S}$ given by Eq. (4.18). In cylindrical coordinates with the $z$ axis along the line joining the two quarks at $z= \pm R / 2$, Eq. (3.10) gives

$$
\vec{D}_{S}=e \hat{e}_{z}\{\theta(z-R / 2)-\theta(z+R / 2)\} \delta(x) \delta(y),
$$

which describes the polarization vector for a line of dipoles. The solution of Eq. (6.14) is

$$
\vec{C}^{D}=\hat{e}_{\phi} C^{D},
$$

where

$$
C^{D}=\frac{e}{4 \pi \rho}\left\{\frac{z-R / 2}{\sqrt{\rho^{2}+(z-R / 2)^{2}}}-\frac{(z+R / 2)}{\sqrt{\rho^{2}+(z+R / 2)^{2}}}\right\} .
$$

Then

$$
\vec{c}=\hat{e}_{\phi} c,
$$

and Eq. (6.10) becomes the following equation for $c$ :

$$
\left(\widetilde{\nabla^{2}}-6 g^{2} B^{2}\right) c=6 g^{2} B^{2} C^{D},
$$

where

$$
\widetilde{\nabla}^{2} f(\rho, z) \equiv \frac{\partial}{\partial \rho}\left(\frac{1}{\rho} \frac{\partial}{\partial \rho}(\rho f)\right)+\frac{\partial^{2} f}{\partial z^{2}} .
$$

Equations (6.11), (6.12), and (6.19) are three nonlinear equations for the static configuration $c, B$, and $B_{3}$ with boundary conditions: $c \rightarrow-C^{D}, B \rightarrow B_{0}, B_{3} \rightarrow B_{0}$ at large distances; $c \rightarrow 0, B \rightarrow 0$ for $\vec{x}$ on $L$. These equations have been solved [19] with the following results:

The monopole current in Eq. (6.10) screens the color electric field produced by the quark sources so that as the quarkantiquark separation increases the lines of $\vec{D}$ are compressed from their Coulomb-like behavior at small $R$ to form a flux tube, and thus $V_{0}(R) \rightarrow \sigma R$ at large $R$. Both this small $R$ and this large $R$ behavior of the potential have their common origin in the evolving distribution of the flux of $\vec{D}$ whose divergence is fixed by the color electric charge of the quarks $\left(\vec{\nabla} \cdot \vec{D}=\vec{\nabla} \cdot \vec{D}_{S}\right)$ and whose curl is determined by the monopole current. Thus, the dual theory already in the classical approximation gives a potential that evolves smoothly from the large $R$ confinement region to the short-distance perturbative domain. This shows how the dual theory realizes the Mandelstam-'t Hooft mechanism. It does not describe QCD at shorter distances where radiative corrections giving rise to asymptotic freedom and a running coupling constant are important.

To calculate the terms in $i \ln W_{\text {eff }}$ that are quadratic in the quark velocities we solve the field equations for moving quarks. To first order in the velocities the static field distributions follow the quark motion adiabatically. The time de- pendence of $\vec{C}, B$, and $B_{3}$ then results from the explicit time dependence of $R$. Furthermore, since $\int d \vec{x} \mathcal{L}_{0}$ generates the static field equations, it is stationary about the solution to these equations and remains unchanged to second order in the velocities. The velocity dependence in the potential then comes from the "magnetic" contribution $\mathcal{L}_{2}$, which depends quadratically upon $\partial_{0} \vec{C}, \partial_{0} B_{3}$, and $C_{0}$, all of which are first order in the velocities. The scalar potential $C_{0}$ satisfies the equation, obtained from the time component of Eq. (6.2),

$$
\nabla^{2} C_{0}-6 g^{2} B^{2} C_{0}=\vec{\nabla} \cdot \vec{H}_{S},
$$

valid to first order in the velocities. With the Higgs field $B(\vec{x})$ already determined by the static equations, Eq. (6.21) is a linear equation for the scalar potential, giving $C_{0}$ to first order in the velocity. The velocity dependent potential $V_{\mathrm{VD}}$ is then given by

$$
V_{\mathrm{VD}}=-\int d \vec{x} \mathcal{L}_{2}
$$

representing the magnetic color energy due to the fields following the moving quarks.

For small $R$ the potential $V_{\mathrm{VD}}$ approaches the velocity dependent part of the Darwin potential (3.7) (multiplied by the color factor $4 / 3$ ) because for small $R$ the color magnetic field $\vec{H}(\vec{x})$ becomes the ordinary Biot-Savart magnetic field. As $R$ increases the color magnetic field lines are compressed so that for large separation $V_{\mathrm{VD}}$ becomes linear in $R$. As an example consider the case in which two equal mass quarks move in a circular orbit of frequency $\omega$. Then $\vec{v}_{1}=-\vec{v}_{2}=(\vec{\omega} \times \vec{R} / 2)$, so that $\vec{\omega}$ is proportional to $1 / R$ and $V_{\mathrm{VD}}$ reduces to

$$
V_{\mathrm{VD}}=-\frac{1}{2} I(R) \omega^{2},
$$

where

$$
\lim _{R \rightarrow \infty} I(R) \sim \frac{1}{2}(A R) R^{2},
$$

and where

$$
A \simeq 0.21 \sigma,
$$

determined numerically [1] from Eq. (6.22). By comparison we note that the moment of inertia $I^{\prime}(R)$ of an infinitely thin flux tube of length $R$ is

$$
I^{\prime}(R)=\frac{1}{2}\left(A^{\prime} R\right) R^{2}
$$

with

$$
A^{\prime}=\sigma / 6 \text {. }
$$

The comparison of Eq. (6.27) describing an infinitely thin flux tube with Eq. (6.25) gives a quantitative estimate of the increase of the moment of inertia $I(R)$ of the flux tube due to its finite thickness. 
We now compare these results for $V_{0}+V_{\mathrm{VD}}=-L_{I}$ of the dual theory with the "modified area law" model [9] Eq. (2.22). In the dual theory $i \ln W(\Gamma)$ is replaced by $i \ln W_{\text {eff }}(\Gamma)$, given in the classical approximation by Eq. (6.1). This gives in the limit of short distances the perturbative expression Eq. (3.7) so that the short-distance limit of the dual theory is the short-range component $i \ln W^{\mathrm{SR}}(\Gamma)$. The long distance limit of $i \ln W_{\text {eff }}(\Gamma)$ is fixed by the values of $\sigma$ and $A$. Replacing $A$ by $A^{\prime}$ in this limit yields $i \ln W^{\mathrm{LR}}(\Gamma)$. This shows that $i \ln W^{\mathrm{LR}}(\Gamma)$ describes a zerowidth flux tube. Aside from this difference we see that the "two components" of Eq. (2.22) arise as two limits of a single classical solution describing the evolution of the potential produced by compression of the field lines with increasing $R$.

As the simplest example of the implications of $V_{\mathrm{VD}}$, we add relativistic kinetic energy terms to $-\left(V_{0}+V_{\mathrm{VD}}\right)$ to obtain a classical Lagrangian, and calculate classically the energy and angular momentum of $q \bar{q}$ circular orbits, which are those that have the largest angular momentum $J$ for a given energy. We find [20] a Regge trajectory $J$ as a function of $E^{2}$ that for large $E^{2}$ becomes linear with slope $\alpha^{\prime}=J / E^{2}=1 / 8 \sigma(1-A / \sigma)$. Then Eq. (6.25) gives $\alpha^{\prime} \approx 1 /(6.3 \sigma)$, which is close to the string model relation $\alpha^{\prime}=1 /(2 \pi \sigma)$. This comparison shows how at the classical level a string model emerges when the velocity dependence of the $q \bar{q}$ potential is included. The fact that the difference between the two expressions for $\alpha^{\prime}$ is small indicates that the infinity narrow string may be a good approximation to the finite-width flux tube forms between the $q \bar{q}$ pair.

To summarize: (1) The potential $V_{0}(R)$ is determined by Eqs. (6.9) and (4.21) evaluated at the static solution. (2) The potential $V_{\mathrm{VD}}$ is given by Eqs. (6.22) and (4.22) evaluated at the solution of the classical equations to first order in the velocity. The resultant integrals have been calculated numerically [1] and determine four functions $V_{+}(R), V_{-}(R), V_{L}(R)$, and $V_{\|}(R)$ that specify uniquely the terms in the potential proportional to the velocity squared. Explicit expressions for these functions are given in Ref. [1].

\section{Remarks}

(1) In the absence of quark sources $\left(G_{\mu \nu}^{S}=0\right), \mathcal{L}_{\text {eff }}$ describes a system of massive dual gluons and monopoles. Because of the dual Higgs boson mechanism there are no unwanted massless particles in the spectrum. The massive particles of the dual theory cannot be identified with the massive particles of Yang-Mills theory, since the dual theory just describes the low-energy spectrum. These masses determine rather the scale $R_{\mathrm{FT}}=1 / M$ above which the dual theory should describe the $q \bar{q}$ interaction. Since a quark-antiquark pair moving in an orbit of radius $R$ can only radiate a particle of mass $M$ if $1 / R>M$, in the domain $R>1 / M$ where the dual theory describes Yang-Mills theory no dual gluons or monopoles are emitted. The glueballs of Yang-Mills theory, on the otherhand, are described by closed loops of color flux, obtained by coupling the dual potentials to closed Dirac strings and finding the corresponding static solution of the field equations of the dual theory.
(2) The Lagrangian density $\mathcal{L}_{\text {eff }}$ (4.17) describes the coupling of the Dirac string to Abelian configurations of dual potentials, and the functional integral (4.23) for $W_{\text {eff }}(\Gamma)$ is restricted to such configurations. The external $q \bar{q}$ pair has in effect selected out a particular sector of the dual theory relevant to the $q \bar{q}$ potential. As a consequence the resulting potential should not be very sensitive to the details of the dual gauge group.

(3) The Dirac string in the classical solution was a straight line connecting the $q \bar{q}$ pair. This gave the configuration having the minimum field energy. ${ }^{4}$ The flux tube corresponding to a given string position is concentrated in the neighborhood of that string since the monopole current vanishes there. To evaluate the contributions to the potential arising from fluctuations of the shape and length of the flux tube [21] we must integrate over field configurations generated by all Dirac strings connecting the $q \bar{q}$ pair. This amounts to doing a functional integral over all Dirac polarization tensors $G_{\mu \nu}^{S}(x)$. Similar integrals have recently been carried out by Akhmedov et al. [10] in a somewhat different context. The functional integral over $G_{\mu \nu}^{S}(x)$ is replaced by a functional integral over corresponding world sheets $y^{\mu}(\sigma, \tau)$, multiplied by an appropriate Jacobian. As a result they obtain [10] an effective string theory free from the conformal anomaly [22]. Such techniques when applied in the context of the dual theory should lead to a corresponding effective string theory.

\section{THE CLASSICAL APPROXIMATION FOR $\boldsymbol{V}_{\text {SD }}$}

In this section we evaluate the expression for $V_{\mathrm{SD}}$ given in Sec. V using the classical solutions to the dual theory described in Sec. VI. We consider separately the four contributions to $V_{\mathrm{SD}}$ [see Eq. (2.8)].

(1) $V_{\text {Thomas }}$ : Equations (5.17) and (5.18) with $V_{0}(R)$ determined by Eq. (6.9).

(2) $V_{\mathrm{LS}}^{\mathrm{mag}}$ : Equation (5.15) with $\langle\langle\vec{D}\rangle\rangle_{\text {eff }}$ and $\langle\langle\vec{H}\rangle\rangle_{\text {eff }}$ replaced by their classical values $\vec{D}$ and $\vec{H}$, namely

$$
V_{\mathrm{LS}}^{\mathrm{mag}}=-\sum_{j=1}^{2} \frac{4}{3} \frac{e_{j}}{m_{j}} \vec{S}_{j} \cdot\left(\vec{H}\left(\vec{z}_{j}\right)-\vec{v}_{j} \times \vec{D}\left(\vec{z}_{j}\right)\right),
$$

with $\vec{H}-\vec{v}_{j} \times \vec{D}$ calculated to first order in the velocity. To this order the static field configurations follow the motion of the quarks adiabatically and we find from, Eq. (4.19),

$$
\vec{H}\left(\vec{z}_{j}\right)-\vec{v}_{j} \times \vec{D}\left(\vec{z}_{j}\right)=-\left.\vec{\nabla}\left(C_{0}(\vec{x})-\vec{C}(\vec{x}) \cdot \vec{v}(\vec{x})\right)\right|_{\vec{x}=\vec{z}_{j}},
$$

where

$$
\vec{v}(\vec{x})=\frac{\vec{v}_{1}+\vec{v}_{2}}{2}+\vec{\omega} \times \vec{x}
$$

\footnotetext{
${ }^{4}$ The Dirac string of the dual theory, in contrast to that of electrodynamics, is physical. The vanishing of the Higgs field on the string produces a vortex and an associated flux tube containing energy. This vortex cannot be removed by a gauge transformation since such a transformation leaves the magnitude of the Higgs field unchanged.
} 
and

$$
\vec{\omega}=\frac{\vec{R} \times(d \vec{R} / d t)}{R^{2}}
$$

In Eqs. (7.2)-(7.4), $\left(\vec{v}_{1}+\vec{v}_{2}\right) / 2$ is the instantaneous velocity of the origin of the coordinates that we have chosen as the midpoint of the line $L$ connecting the $q \bar{q}$ pair and $\vec{\omega}$ is the instantaneous angular velocity of $L$. [The motion of the $q \bar{q}$ pair along $L$ does not contribute to Eq. (7.2)].

We can understand the result (7.2), as follows. The lefthand side is the color magnetic field at the position of the $j$ th quark in the Lorentz system in which it is instantaneously at rest. The magnetic field in this comoving system is determined by the gradient of the corresponding dual scalar potential, namely $C_{0}-\vec{C} \cdot \vec{v}$. Indeed Eq. (7.2) remains valid beyond the classical approximation with the replacement $C_{0} \rightarrow\left\langle\left\langle C_{0}\right\rangle\right\rangle_{\text {eff }}, \quad \vec{C} \rightarrow\langle\langle\vec{C}\rangle\rangle_{\text {eff }}$.

Choosing $\vec{R}$ to lie along the $z$ axis and using Eqs. (6.21) and (6.10) for $C_{0}$ and $\vec{C}$ we find

$$
C_{0}-\vec{C} \cdot \vec{v}=\hat{e}_{\phi} \cdot \frac{d \vec{R}}{d t} C_{-}(z, \rho),
$$

where $\rho, \phi, z$ are cylindrical coordinates, and

$$
C_{-}(z, \rho)=C_{-}^{D}(z, \rho)+c_{-}(z, \rho),
$$

where

$$
C_{-}^{D}(z, \rho)=\frac{e \rho}{4 \pi R}\left\{\frac{1}{\sqrt{\rho^{2}+(z-R / 2)^{2}}}-\frac{1}{\sqrt{\rho^{2}+(z+R / 2)^{2}}}\right\},
$$

and where $c_{-}(z, \rho)$ satisfies the equation

$$
\left(\widetilde{\nabla}^{2}-6 g^{2} B^{2}\right) c_{-}=6 g^{2} B^{2} C_{-}^{D}-\frac{2}{R} \frac{\partial c}{\partial z}
$$

The solution of the linear integral equation (7.8) for $c_{-}$ determines, via Eqs. (7.2) and (7.5) the nonperturbative part of the color magnetic field in the comoving Lorentz system. From Eqs. (7.6)-(7.8) it follows that for any fixed value of $z$ and $\rho$ this field vanishes like $1 / R$ for large $q \bar{q}$ separation. The vanishing of this field at large $R$ is in accordance with the observation of Buchmuller [23] that in a flux tube picture the color field in the comoving frame should be purely electric. However, for any finite value of the $q \bar{q}$ separation there is a color magnetic field in this system, and Eqs. (7.1)-(7.8) give

$$
\begin{aligned}
V_{\mathrm{LS}}^{\mathrm{mag}}= & \frac{V_{2}^{\prime}(R)}{R}\left\{\left(\frac{\vec{S}_{1} \cdot\left(\vec{R} \times \vec{p}_{1}\right)}{m_{1}^{2}}-\frac{\vec{S}_{2} \cdot\left(\vec{R} \times \vec{p}_{2}\right)}{m_{2}^{2}}\right)\right. \\
& \left.+\left(\frac{\vec{S}_{2} \cdot\left(\vec{R} \times \vec{p}_{1}\right)}{m_{1} m_{2}}-\frac{\vec{S}_{1} \cdot\left(\vec{R} \times \vec{p}_{2}\right)}{m_{1} m_{2}}\right)\right\},
\end{aligned}
$$

where

$$
V_{2}^{\prime}(R)=\frac{4}{3}\left\{\frac{\alpha_{s}}{R^{2}}-\left.\frac{e}{2 \rho} \frac{\partial}{\partial \rho}\left[\rho c_{-}(\rho, z)\right]\right|_{\substack{\rho=0 \\ z=R / 2}}\right\}
$$

The first term in Eq. (7.10) is the perturbative contribution to $V_{2}^{\prime}(R)$ arising from $C_{-}^{D}$ and the second term is the nonperturbative part that behaves like $1 / R$ for large $R$ and that would not be present in the simple flux tube picture of Buchmuller.

Finally adding $V_{\mathrm{LS}}^{\mathrm{mag}}$ to $V_{\text {Thomas }}$ gives the complete expression for the spin orbit coupling $V_{\mathrm{LS}}$ :

$$
\begin{aligned}
V_{\mathrm{LS}}= & {\left[\frac{1}{R} \frac{d V_{0}}{d R}+2 \frac{V_{1}^{\prime}(R)}{R}\right]\left[\frac{\vec{S}_{1} \cdot \vec{R} \times \vec{p}_{1}}{2 m_{1}^{2}}-\frac{\vec{S}_{2} \cdot \vec{R} \times \vec{p}_{2}}{2 m_{2}^{2}}\right] } \\
& +\frac{V_{2}^{\prime}(R)}{R}\left[\frac{\vec{S}_{2} \cdot \vec{R} \times \vec{p}_{1}}{m_{1} m_{2}}-\frac{\vec{S}_{1} \cdot \vec{R} \times \vec{p}_{2}}{m_{1} m_{2}}\right]
\end{aligned}
$$

where

$$
V_{1}^{\prime}(R)=V_{2}^{\prime}(R)-\frac{d V_{0}}{d R}
$$

Equation (7.11) expresses the spin-orbit potential in terms of the static potential and a single independent function $V_{2}^{\prime}(R)$ determined by the dual scalar potential $C_{0}-\vec{C} \cdot \vec{v}$ in the comoving frame. This result for $V_{\mathrm{LS}}$ satisfies identically the constraints of Lorentz invariance (7.12) (the Gromes relations [24]). Furthermore, since $V_{2}^{\prime}(R) \rightarrow 1 / R$ for large $R$, we have

$$
\lim _{R \rightarrow \infty} V_{1}^{\prime}(R) \sim-\frac{d V_{0}}{d R}=-\sigma
$$

which is the value given by the flux tube model for all $R$.

(3) $V_{\mathrm{SS}}$ : Equation (5.22) with $\delta\left\langle\left\langle\vec{H}\left(z_{j}\right)\right\rangle\right\rangle_{\mathrm{eff}} / \delta \vec{H}_{S}\left(z_{j^{\prime}}\right)$ replaced by $\delta \vec{H}\left(z_{j}\right) / \delta \vec{H}_{S}\left(z_{j^{\prime}}\right)$. Since, to first order in the velocity, $\vec{C}$ is determined by $\vec{D}_{S}$ alone [see Eq. (6.10)] the $\partial \vec{C} / \partial t$ term in $\vec{H}$ does not contribute to its variational derivative with respect to $\vec{H}_{S}$ and Eq. (4.19) gives

$$
\frac{\delta H_{k}(x)}{\delta H_{S}\left(x^{\prime}\right)}=\delta_{k \ell} \delta\left(\vec{x}-\vec{x}^{\prime}\right) \delta\left(t-t^{\prime}\right)-\nabla_{k} \frac{\delta C_{0}(x)}{\delta H_{S}\left(x^{\prime}\right)} \text {. }
$$

The quantity $\delta C_{0} / \delta \vec{H}_{S}$ in turn satisfies the equation obtained by taking the variational derivative of Eq. (6.21) with respect to $\vec{H}_{S}$ : namely,

$$
\left(\nabla^{2}-6 g^{2} B^{2}\right) \frac{\delta C_{0}(x)}{\delta H_{S}\left(x^{\prime}\right)}=\nabla / \delta\left(\vec{x}-\vec{x}^{\prime}\right) \delta\left(t-t^{\prime}\right) .
$$

The double integral in Eq. (5.22) then becomes a single integral over $t$ of the static quantity $\delta \vec{H}\left(\vec{z}_{j}\right) / \delta \vec{H}_{S}\left(\vec{z}_{j^{\prime}}\right)$. We emphasize that this simplification obtains only in the classical approximation we are now considering.

Equations (7.14) and (7.15) give 


$$
\frac{\delta H_{k}(\vec{x})}{\delta H_{S \ell}\left(\vec{x}^{\prime}\right)}=\delta_{k \ell} \delta\left(\vec{x}-\vec{x}^{\prime}\right)+\nabla_{k} \nabla_{\ell}^{\prime} G\left(\vec{x}, \vec{x}^{\prime}\right),
$$

where the Green function $G\left(\vec{x}, \vec{x}^{\prime}\right)$ satisfies

$$
\left(-\nabla^{2}+6 g^{2} B^{2}(\vec{x})\right) G\left(\vec{x}, \vec{x}^{\prime}\right)=\delta\left(\vec{x}-\vec{x}^{\prime}\right) .
$$

$G\left(\vec{x}, \vec{x}^{\prime}\right)$ is the potential at $\vec{x}$ due to a point charge at $\vec{x}^{\prime}$ in presence of the monopole charge density $j_{0}^{\text {mon }}$ (6.8) carried by $B(\vec{x})$. Since $B(\vec{x})$ approaches its vacuum value $B_{0}$ as $\vec{x} \rightarrow \infty, G$ vanishes exponentially at large distances: i.e.,

$$
G\left(\vec{x}, \vec{x}^{\prime}\right) \underset{x \rightarrow \infty}{\sim}-\frac{e^{-M\left|\vec{x}-\vec{x}^{\prime}\right|}}{4 \pi\left|\vec{x}-\vec{x}^{\prime}\right|},
$$

where $M$ is the mass of the dual gluon,

$$
M^{2}=6 g^{2} B_{0}^{2}=\frac{6 \pi}{\alpha_{s}} B_{0}^{2} \approx \frac{\pi}{4} \frac{\sigma}{\alpha_{s}}
$$

and where we used the result, $\sigma \approx 24 B_{0}^{2}$, obtained from the energy per unit length of the static flux tube solution. Using a value $\alpha_{s}=0.37$ obtained from fitting the $c \bar{c}$ and $b \bar{b}$ spectrum [1] we obtain $M \approx 640 \mathrm{MeV}$.

Separating off the Coulomb contribution to $G$ we have

$$
G=-\frac{1}{4 \pi\left|\vec{x}-\vec{x}^{\prime}\right|}+G^{\mathrm{NP}}
$$

where $G^{N P}$ satisfies the equation

$$
\left(-\nabla^{2}+6 g^{2} B^{2}\right) G^{\mathrm{NP}}=-\frac{6 g^{2} B^{2}(\vec{x})}{4 \pi\left|\vec{x}-\vec{x}^{\prime}\right|} .
$$

Inserting Eqs. (7.16) and (7.20) into Eq. (5.22) gives

$$
V_{\mathrm{SS}}=V_{\mathrm{SS}}^{\mathrm{spin}}+V_{\mathrm{SS}}^{1 / m^{2}}
$$

where

$$
\begin{aligned}
V_{\mathrm{SS}}^{\mathrm{spin}}= & \frac{4}{3} \frac{e^{2}}{m_{1} m_{2}}\left\{\left(\vec{S}_{1} \cdot \vec{S}_{2}\right) \delta\left(\vec{z}_{1}-\vec{z}_{2}\right)\right. \\
& +\left.\left(\vec{S}_{1} \cdot \vec{\nabla}\right)\left(\vec{S}_{2} \cdot \vec{\nabla}^{\prime}\right) G\left(\vec{x}, \vec{x}^{\prime}\right)\right|_{\left.\vec{x}=\vec{z}_{1}, \vec{x}^{\prime}=\vec{z}_{2}\right\},} \\
V_{\mathrm{SS}}^{1 / m^{2}}= & -\left.\frac{4}{3} \sum_{j=1}^{2} \frac{e^{2}}{8 m_{j}^{2}} \vec{\nabla} \cdot \vec{\nabla}^{\prime} G^{\mathrm{NP}}\left(\vec{x}, \vec{x}^{\prime}\right)\right|_{\vec{x}^{\prime}=\vec{x}=\vec{z}_{j},}
\end{aligned}
$$

The potential $V_{\mathrm{SS}}^{\mathrm{spin}}$ is the same as previously obtained [11]. At small $R$ it approaches the usual perturbative spin-spin interaction, and at long distances it is exponentially damped due to screening by the monopole charge. The spinindependent contribution $V_{\mathrm{SS}}^{1 / m^{2}}$ of $V_{\mathrm{SS}}$ depends upon $R$ via the dependence in Eq. (7.21) of $G^{N P}$ on $B$. It was not included in Ref. [11].

(4) $V_{\text {Darwin }}$ : Equation (5.24) with $\left\langle\left\langle\hat{G}_{\mu \nu}\right\rangle\right\rangle_{\text {eff }}$ replaced by $\hat{G}_{\mu \nu}$, namely,

$$
\int V_{\text {Darwin }} d t=-\frac{4}{3} \sum_{j} \frac{e}{8 m_{j}^{2}} \int_{\Gamma_{j}} d x^{\mu} \partial^{\nu} \hat{G}_{\nu \mu}(x) .
$$

To evaluate Eq. (7.25) we note from Eqs. (3.11) and (4.16) that

$$
\partial^{\nu} \hat{G}_{\nu \mu}(x)=j_{\mu}(x),
$$

where $j_{\mu}(x)$ is the quark-antiquark current. The monopole current does not contribute to $\partial^{\nu} \hat{G}_{\nu \mu}$ and $V_{\text {Darwin }}$ becomes

$$
\begin{aligned}
\int V_{\text {Darwin }} d t & =-\frac{4}{3} \sum_{j} \frac{e}{8 m_{j}^{2}} \int_{\Gamma_{j}} d x^{\mu} j_{\mu}(x) \\
& =-\frac{4}{3} \sum_{j} \frac{e_{j}}{8 m_{j}^{2}} \int d t \rho\left(z_{j}\right) .
\end{aligned}
$$

Omitting self-energy terms we insert $\rho\left(\vec{z}_{1}\right)$ $=-e \delta\left(\vec{z}_{1}-\vec{z}_{2}\right), \rho\left(\vec{z}_{2}\right)=e \delta\left(\vec{z}_{2}-\vec{z}_{1}\right)$ into Eq. (7.27) and obtain

$$
V_{\text {Darwin }}=\frac{e^{2}}{6}\left(\frac{1}{m_{1}^{2}}+\frac{1}{m_{2}^{2}}\right) \delta\left(\vec{z}_{1}-\vec{z}_{2}\right) .
$$

In Appendix B we show that the alternate form (B3) for $V_{\text {Darwin }}$ reduces in the classical approximation to the same expression (7.28).

There are two then spin-independent terms proportional to $\left(1 / m_{1}^{2}+1 / m_{2}^{2}\right)$. The first is $V_{\mathrm{SS}}^{1 / m^{2}}(7.24)$. The second is $V_{\text {Darwin }}$ (7.28).

To summarize: In Ref. [4] the coefficient of $\left(1 / m_{1}^{2}+1 / m_{2}^{2}\right)$ in the velocity dependent potential was written as

$$
V_{\mathrm{SS}}^{1 / m^{2}}+V_{\text {Darwin }} \equiv \frac{1}{8}\left(\frac{1}{m_{1}^{2}}+\frac{1}{m_{2}^{2}}\right) \nabla^{2}\left(V_{0}(R)+V_{a}(R)\right)
$$

which defines $V_{a}$. Equations (7.24) and (7.28) give

$$
\nabla^{2} V_{a}=\nabla^{2} V_{0}^{\mathrm{NP}}(R)-\left.\frac{4}{3} e^{2} \vec{\nabla} \cdot \vec{\nabla}^{\prime} G^{\mathrm{NP}}\left(\vec{x}, \vec{x}^{\prime}\right)\right|_{\vec{x}=\vec{x}^{\prime}=\vec{z}_{j},}
$$

where $V_{0}^{\mathrm{NP}}(R)$ is the nonperturbative part of the static potential so that $V_{a}$ is determined by the nonperturbative dynamics of Yang-Mills theory. The first term in Eq. (7.30) is the color electric contribution to $V_{a}$ and the second is the color magnetic contribution.

The spin dependent potential is then given by

$$
V_{\mathrm{SD}}=V_{\mathrm{LS}}+V_{\mathrm{SS}}^{\mathrm{spin}}+\frac{1}{8}\left(\frac{1}{m_{1}^{2}}+\frac{1}{m_{2}^{2}}\right) \nabla^{2}\left[V_{0}(R)+V_{a}(R)\right] \text {, }
$$

with $V_{\text {LS }}$ given by Eq. (7.10) and (7.11), $V_{\mathrm{SS}}^{\text {spin }}$ by Eq. (7.23) and $V_{a}(R)$ by Eq. (7.30).

It should be emphasized that to account for the effect of fluctuations of the flux tube on the potential we must return 
to Eqs. (5.15), (5.22), (5.24), and (5.3) that express $V_{q \bar{q}}$ in terms of dual potentials, independent of the classical approximation.

\section{CONCLUSION}

We have obtained an expression for the heavy quark potential $V_{q \bar{q}}$ in terms of an effective Wilson loop $W_{\text {eff }}(\Gamma)$ determined by the dynamics of a dual theory that is weakly coupled at long distances. The coupling of the dual variables to heavy quarks is then uniquely specified, with spin and relativistic effects accounted for unambiguously to order $(1 / \text { mass quark })^{2}$, the highest order for which the concept of a potential makes sense.

The classical approximation gives the leading longdistance contribution to $W_{\text {eff }}(\Gamma)$ and yields a velocitydependent spin-dependent heavy quark potential that for large $R$ becomes linear in $R$ and that for small $R$ approaches lowest-order perturbative QCD. The dual theory cannot describe QCD at shorter distances, where radiative corrections giving rise to asymptotic freedom become important. At such distances the dual potentials are strongly coupled and the dual description is no longer appropriate.

As a final remark we note that the dual theory is an SU(3) gauge theory, like the original Yang-Mills gauge theory. However, the coupling to quarks selected out only Abelian configurations of the dual potential. Therefore, our results for the $q \bar{q}$ interaction do not depend upon the details of the dual gauge group and should be regarded more as consequences of the general dual superconductor picture rather than of our particular realization of it. The essential feature of this picture is the description of long-distance Yang-Mills theory by a dual gauge theory in which all particles become massive via a dual Higgs mechanism.

\section{ACKNOWLEDGMENTS}

One of us (M.B) would like to thank M. Polikarpov and L. Yaffe for enlightening conversations. The work of M.B. was supported in part by the U.S. Department of Energy under Grant No. DOE/ER/40614. The work of F.Z. was supported in part by the U.S. Department of Energy under Grant No. DE-FG03-92-ER40701.

\section{APPENDIX A}

Notice that

$$
\vec{a}_{j} \equiv \frac{4}{3} \frac{e_{j}}{m_{j}}\left\langle\left\langle\vec{D}\left(\vec{z}_{j}\right)\right\rangle\right\rangle_{\mathrm{eff}}
$$

can be interpreted as the acceleration of the $j$ th quark so that Eq. (5.17) can be rewritten

$$
V_{\text {Thomas }}=-\frac{1}{2} \sum_{j=1}^{2} S_{j} \cdot\left(\vec{v}_{j} \times \vec{a}_{j}\right)
$$

which is the usual expression obtained from semiclassical considerations. To express $V_{\text {Thomas }}$ in terms of the derivative of the static potential we first note from Eq. (5.9) that

$$
\frac{\delta i \ln W_{\mathrm{eff}}(\Gamma)}{\delta \vec{D}_{S}(\vec{x})}=\frac{4}{3}\langle\langle\vec{D}(\vec{x})\rangle\rangle_{\mathrm{eff}} .
$$

Now, using the fact that

$$
\vec{\nabla}_{1} \vec{D}_{S}(\vec{x})=-e \mathbf{1} \delta\left(\vec{x}-\vec{z}_{1}\right)
$$

where $\vec{\nabla}_{1}=\partial / \partial \vec{z}_{1}$, we have

$$
\begin{aligned}
R \frac{d V_{0}(R)}{d R} & =\vec{R} \cdot \vec{\nabla}_{1} V_{0} \\
& =\vec{R} \int d \vec{x} \frac{\delta i \ln W_{\mathrm{eff}}}{\delta \vec{D}_{S}(\vec{x})} \cdot \vec{\nabla}_{1} \vec{D}_{S}(\vec{x}) \\
& =-e \vec{R} \cdot \int d \vec{x} \frac{4}{3}\langle\langle\vec{D}(\vec{x})\rangle\rangle_{\mathrm{eff}} \delta\left(\vec{x}-\vec{z}_{1}\right) \\
& =-e \frac{4}{3} R\left\langle\left\langle\vec{D}\left(\vec{z}_{1}\right)\right\rangle\right\rangle_{\mathrm{eff}} \cdot \hat{R} .
\end{aligned}
$$

Now by symmetry, $\left\langle\left\langle\vec{D}\left(\vec{z}_{1}\right)\right\rangle\right\rangle_{\text {eff }}$ evaluated at the position of a quark must lie along $\hat{R}$. Hence,

$$
-\frac{4}{3} e\left\langle\left\langle\vec{D}\left(\vec{z}_{1}\right)\right\rangle\right\rangle_{\mathrm{eff}}=\hat{R} \frac{d V_{0}}{d R} .
$$

Equation (A1) then gives $\vec{a}_{1}=-\left(\hat{R} / m_{1}\right)\left(\partial V_{0} / \partial R\right)$, so that Eq. (A2) gives Eq. (5.18).

\section{APPENDIX B}

Here we begin with an alternate form for $V_{\text {Darwin }}$ where $A_{\mu}$ does not appear explicitly.

$$
\begin{aligned}
e \int & d x^{\mu}\left\langle\left\langle D^{\nu} F_{\nu \mu}(x)\right\rangle\right\rangle \\
= & \int_{t_{i}}^{t_{f}} d t \nabla^{2} V_{0}-i e^{2} \int_{\Gamma_{j}} d x^{\mu} \int_{\Gamma_{j}} d x_{\sigma}^{\prime}\left[\left\langle\left\langle F_{\mu \nu}(x) F^{\sigma \nu}\left(x^{\prime}\right)\right\rangle\right\rangle\right. \\
& \left.-\left\langle\left\langle F_{\mu \nu}(x)\right\rangle\right\rangle\left\langle\left\langle F^{\sigma \nu}\left(x^{\prime}\right)\right\rangle\right\rangle\right] \\
= & \int_{t_{i}}^{t_{f}} d t \nabla^{2} V_{0}-i e^{2} \int_{\Gamma_{j}} d t \int_{\Gamma_{j}} d t^{\prime}\left[\left\langle\left\langle F_{0 k}\left(z_{j}\right) F^{0 k}\left(z_{j}^{\prime}\right)\right\rangle\right\rangle\right. \\
& \left.-\left\langle\left\langle F_{0 k}\left(z_{j}\right)\right\rangle\right\rangle\left\langle\left\langle F^{0 k}\left(z_{j}^{\prime}\right)\right\rangle\right\rangle\right] .
\end{aligned}
$$

Equation (5.12) and the relation between $\delta S^{\mu \nu}$ and variations of $G_{\mu \nu}^{S}$ give

$$
\begin{gathered}
e \int_{\Gamma_{j}} d t \int_{\Gamma_{j}} d t^{\prime} \frac{\delta}{\delta S^{0 k}\left(z_{j}^{\prime}\right)}\left\langle\left\langle F^{0 k}\left(z_{j}\right)\right\rangle\right\rangle \\
=\frac{4 e^{2}}{3} \int_{\Gamma_{j}} d t \int_{\Gamma_{j}} d t^{\prime} \frac{\delta\left\langle\left\langle D_{k}\left(z_{j}\right)\right\rangle\right\rangle_{\mathrm{eff}}}{\delta D_{S k}\left(z_{j}^{\prime}\right)} .
\end{gathered}
$$

Then using Eq. (2.21) with $z_{2}$ replaced by $z_{1}$ and $e$ by $-e$ we obtain 


$$
\begin{aligned}
\int d t V_{\text {Darwin }}= & \sum_{j} \int d t \nabla^{2} V_{0} / 8 m_{j}^{2} \\
& -\frac{4 e^{2}}{3} \sum_{j} \int_{\Gamma_{j}} d t \int_{\Gamma_{j}} d t^{\prime} \frac{1}{8 m_{j}^{2}} \frac{\delta\left\langle\left\langle D_{k}\left(z_{j}\right)\right\rangle\right\rangle_{\mathrm{eff}}}{\delta D_{S k}\left(z_{j}^{\prime}\right)},
\end{aligned}
$$

which gives a second form for $V_{\text {Darwin }}$. The classical approximation to Eq. (B3) is obtained by replacing

$$
\frac{\delta\left\langle\left\langle\vec{D}\left(z_{j}\right)\right\rangle\right\rangle_{\mathrm{eff}}}{\delta \vec{D}_{S}\left(z_{j}^{\prime}\right)} \text { by } \frac{\delta \vec{D}\left(\vec{z}_{j}\right)}{\delta \vec{D}_{S}\left(\vec{z}_{j}\right)} \delta\left(t-t^{\prime}\right) .
$$

This yields the expression

$$
V_{\text {Darwin }}=\sum_{j=1}^{2}\left[\frac{1}{8 m_{j}^{2}} \nabla^{2} V_{0}(R)-\frac{4}{3} \frac{e^{2}}{8 m_{j}^{2}} \frac{\delta D_{k}\left(\vec{z}_{j}\right)}{\delta D_{S k}\left(\vec{z}_{j}\right)}\right]
$$

Following the same reasoning that led to Eq. (A5) we obtain

$$
\frac{4}{3} e^{2} \frac{\delta D_{k}\left(\vec{z}_{1}\right)}{\delta D_{S k}\left(\vec{z}_{1}\right)}=\nabla_{1}^{2} V_{0}^{\mathrm{NP}}(R)
$$

[There is no perturbative contribution to the left-hand side of Eq. (B5).] The second term in Eq. (B4) then cancels the nonperturbative part of the first term. Equation (B4) then becomes

$$
\begin{aligned}
V_{\text {Darwin }} & =\left(\frac{1}{8 m_{1}^{2}}+\frac{1}{8 m_{2}^{2}}\right) \nabla^{2}\left(-\frac{4}{3} \frac{e^{2}}{4 \pi R}\right) \\
& =e^{2} \frac{\delta\left(\vec{z}_{1}-\vec{z}_{2}\right)}{6}\left(\frac{1}{m_{1}^{2}}+\frac{1}{m_{2}^{2}}\right),
\end{aligned}
$$

which coincides with Eq. (7.28).
[1] M. Baker, James S. Ball, and F. Zachariasen, Phys. Rev. D 51, 1968 (1995).

[2] N. Brambilla, P. Consoli, and G. M. Prosperi, Phys. Rev. D 50, 5878 (1994).

[3] A. Barchielli, N. Brambilla, and G. M. Prosperi, Nuovo Cimento A 103, 59 (1990).

[4] A. Barchielli, E. Montaldi, and G. M. Prosperi, Nucl. Phys. B296, 625 (1988); B303, 752(E) (1988).

[5] E. Eichten and F. Feinberg, Phys. Rev. D 23, 2724 (1981); M. A. Peskin, in Dynamics and Spectroscopy at High Energy, Proceedings of the 11th Stanford Linear Accelerator Center Summer Institute, Stanford, California, 1983, edited by P. McDonough (SLAC Report No. 207, Stanford, 1984), p. 151.

[6] W. Lucha, F. Schoeberl, and D. Gromes, Phys. Rep. 200, 127 (1991).

[7] N. Seiberg and E. Witten, Nucl. Phys. B426, 19 (1994); B431, 484 (1994).

[8] N. Seiberg, Nucl. Phys. B435, 129 (1995).

[9] N. Brambilla and G. M. Prosperi, in Quark Confinement and the Hadron Spectrum, edited by N. Brambilla and G. M. Prosperi (World Scientific, Singapore, 1995), p. 195; Phys. Lett. B 236, 69 (1990).

[10] E. T. Akhmedov, M. N. Chernodub, M. I. Polykarpov, and M. A. Zubkov, Phys. Rev. D 53, 2087 (1996).

[11] M. Baker, J. S. Ball, and F. Zachariasen, Phys. Rev. D 44, 3949 (1991); Phys. Lett. B 283, 360 (1992).
[12] For another dual theory, see S. Maedan and T. Suzuki, Prog. Theor. Phys. 81, 229 (1989).

[13] L. D. Landau and E. M. Lifshitz, Classical Theory of Fields (Pergamon, New York, 1975), p. 149.

[14] G. G. Darwin, Philos. Mag. 39, 537 (1920).

[15] P. A. M. Dirac, Phys. Rev. 74, 817 (1948).

[16] S. Mandelstam, Phys. Rep. 23C, 145 (1976); G. 't Hooft, in Proceedings of the European Physics Society 1975, edited by A. Zichichi (Editrice Compositori, Bologna, 1976), p. 1225.

[17] M. Baker, J. S. Ball, and F. Zachariasen, Phys. Rev. D 41, 2612 (1990).

[18] A. A. Abrikosov, Sov. Phys. JETP 32, 1442 (1957); H. B. Nielsen and P. Olesen, Nucl. Phys. B61, 45 (1973).

[19] M. Baker, J. S. Ball, and F. Zachariasen, Phys. Rev. D 44, 3328 (1991).

[20] M. Baker, in Proceedings of the Workshop on Quantum Infrared Physics, edited by H. M. Fried and B. Muller (World Scientific, Singapore, 1995), p. 351.

[21] The leading correction to the static potential of $V_{0}(R)$ has the universal value $-\pi / 12 R$. [See M. Luscher, Nucl. Phys. B180, 317 (1981)].

[22] A. M. Polyakov, Phys. Lett. 103B, 207 (1981); 103B, 211 (1981).

[23] W. Buchmuller, Phys. Lett. 112B, 749 (1982).

[24] D. Gromes, Z. Phys. C 26, 401 (1984). 